\title{
Intravenous Administration of Bone Marrow-Derived Mesenchymal Stem Cells Induces a Switch from Classical to Atypical Symptoms in Experimental Autoimmune Encephalomyelitis
}

\author{
Mónica Kurte, ${ }^{1}$ Javiera Bravo-Alegría, ${ }^{1}$ Alexander Torres, ${ }^{1}$ \\ Vania Carrasco, ${ }^{1}$ Cristina Ibáñez, ${ }^{1}$ Ana María Vega-Letter, ${ }^{1}$ \\ Catalina Fernández-O'Ryan, ${ }^{1}$ Carlos E. Irarrázabal, ${ }^{2}$ Fernando E. Figueroa, ${ }^{1}$ \\ Rodrigo A. Fuentealba, ${ }^{1}$ Claudia Riedel, ${ }^{3}$ and Flavio Carrión ${ }^{1}$ \\ ${ }^{1}$ Laboratory of Cellular and Molecular Immunology, Faculty of Medicine, University of Los Andes, 750000 Santiago, Chile \\ ${ }^{2}$ Laboratory of Integrative and Molecular Physiology, Faculty of Medicine, University of Los Andes, 750000 Santiago, Chile \\ ${ }^{3}$ Department of Biological Sciences, Faculty of Biological Sciences, Andrés Bello National University, 8370146 Santiago, Chile
}

Correspondence should be addressed to Flavio Carrión; fcarrion@uandes.cl

Received 13 November 2014; Revised 23 January 2015; Accepted 23 January 2015

Academic Editor: Gary E. Lyons

Copyright @ 2015 Mónica Kurte et al. This is an open access article distributed under the Creative Commons Attribution License, which permits unrestricted use, distribution, and reproduction in any medium, provided the original work is properly cited.

\begin{abstract}
Potent immunosuppressive and regenerative properties of mesenchymal stem cells (MSCs) position them as a novel therapy for autoimmune diseases. This research examines the therapeutic effect of MSCs administration at different disease stages in experimental autoimmune encephalomyelitis (EAE). Classical and atypical scores of EAE, associated with Thl and Th17 response, respectively, and also Treg lymphocytes, were evaluated. MSCs administration at the onset $\left(\mathrm{EAE}+\mathrm{MSC}_{\text {onset }}\right)$ induced an important amelioration of the clinical signs and less lasting effect at the peak of EAE $\left(\mathrm{EAE}+\mathrm{MSC}_{\text {peak }}\right)$. No effect was observed when MSCs were applied after EAE stabilization (EAE $+\mathrm{MSC}_{\text {late }}$ ). Surprisingly, EAE atypical signs were detected in $\mathrm{EAE}+\mathrm{MSC}_{\text {peak }}$ and $\mathrm{EAE}+\mathrm{MSC}_{\text {late }}$ mice. However, no correlation was found in Th17/Th1 ratio. Interestingly, regardless of time administration, MSCs significantly reduced IL-6 and also T-bet, ROR $\gamma$ T, and Foxp3 mRNA levels in brain samples of EAE mice. The downregulation of IL-6 could restore the well-functioning of the blood-brain barrier of EAE mice, correlated with a decreased number of brain infiltrating leukocytes. These results suggest that the inflammatory status is important to be considered for administering MSCs in autoimmune pathologies, leading to a further research to clarify the effect of MSCs for multiple sclerosis.
\end{abstract}

\section{Introduction}

Mesenchymal stem cells (MSCs) are adherent, undifferentiated, pluripotent, and nonhematopoietic progenitor cells principally located in bone marrow and adipose tissue, among others $[1,2]$. In vitro expanded MSCs have the potential to differentiate into mesodermal lineages including osteoblasts, chondrocytes, and adipocytes. Furthermore, mounting evidence demonstrates usefulness for the use of MSCs in tissue repair [3, 4]. Currently, MSCs are also known for their ability to regulate the immune system. The first reports in 2002-2003 showed that MSCs are able to inhibit
T cell proliferation by cell cycle arrest in G0-G1 phase [57]. Today, it is well accepted that MSCs have important immunosuppressive properties over the entire immune system, mainly exerting their effects on $\mathrm{T}, \mathrm{B}, \mathrm{NK}$, and dendritic cells $[8,9]$.

This immunomodulatory capacity of MSCs has opened new therapeutic prospects in the management of proinflammatory and autoimmune pathologies [10-12]. The therapeutic potential of MSCs has been demonstrated in a variety of autoimmune disease models including graftversus-host disease (GVDH) [13], experimental autoimmune encephalomyelitis (EAE) [14, 15], collagen-induced arthritis 
(CIA) [16, 17], MRL/lpr lupus-prone mice [18], and murine model of autoimmune myocarditis [19]. However, treatments with MSCs in these experimental models did not always translate into a decrease of the disease phenotype as expected. In some cases, no beneficial effects were observed during the course of the disease, whereas others demonstrated an exacerbation of clinical symptoms. Zappia et al. showed that MSCs administration was able to substantially reduce the clinical score of EAE animals but only when MSC injection was carried out before the onset [14]. Likewise, Djouad et al. observed that treatment with MSCs conferred no benefit on arthritic animals; and they showed exacerbation of symptoms when MSCs were infused at later stage of the disease [16]. These authors suggested that MSCs were associated with an increase of Th1 response and established that timing of MSCs application is important for MSC-mediated immunosuppression in vivo.

In our previous studies we showed that MSCs inhibit the activation and differentiation of $\mathrm{T}$ helper lymphocytes type 1 (Th1) [20]. Unexpectedly, we also observed that MSCs were able to promote the expansion of Th17 lymphocytes when cocultured with previously activated CD4+ T cells. The opposite effect was observed in Th1 response [20]. Likewise, Guo et al. reported that human MSCs were able to induce Th17 cells, but not Th1 in cocultures of MSCs with naive CD4+ T cells [21]. These data suggest that the MSCs have the capability to generate an imbalance on Th1/Th17 ratio and therefore to change the phenotype of the immune response in vivo.

Multiple sclerosis (MS) is a chronic inflammatory disease characterized by the infiltration of immune system cells to the central nervous system (CNS), causing damage to neuronal axons by loss of myelin [22]. Several studies, using the MS animal model experimental autoimmune encephalomyelitis (EAE), clearly showed that recruitment of $\mathrm{T}$ helper lymphocytes to the CNS is essential for the initiation of the disease [23]. Initially, Th1 lymphocytes were attributed as being primarily responsible for the demyelination of neurons $[24,25]$. However Korn et al. showed that Th17 lymphocytes would be important both at the beginning and at the peak of the disease and possibly Treg lymphocytes at later stages [26]. Moreover, it has been reported that MOG (myelin oligodendrocyte glycoprotein) specific, highly pathogenic Th1 lymphocytes are necessary to allow Th17 entry into the CNS [27]; and it has been suggested that the proportion between Th1 and Th17 determines the site where inflammation occurs in the CNS [28]. Recent findings suggest that Th1 and Th17 lymphocytes generate different clinical signs of EAE. Th1 lymphocytes preferentially induce classic signs of the disease, defined as progressive paralysis of the limbs, from tail to head. On the other hand, Th17 lymphocytes induce atypical symptoms of EAE, characterized by an inability to control coordinate movements and ataxia $[28,29]$. Despite divergent Th1- and Th17-induced pathologies, the in vivo interplay between these pathogenic lymphocyte subtypes appears to be highly complex. Although additional research is required to clearly define the individual contribution of Th1 and Th17 cells in MS, the existence of a distinct phenotype for MOGresponsive Thelper lymphocytes makes the EAE model ideal to study the switch from Th1 to Th17 pathology induced by MSCs.

The aim of this study was twofold. Firstly, it attempts to clarify the therapeutic effect of MSCs injections associated with different degrees of inflammatory conditions using the EAE model. Secondly, because we have previously demonstrated that MSCs are able to promote in vitro Th17 lymphocytes in a proinflammatory context [20], we evaluated if MSCs induce the appearance of atypical signs of EAE associated with an increase on Th17 lymphocytes. Our data indicate that early treatment with MSCs is highly effective in reducing the clinical score of EAE. We propose that the increase in plasma cytokine levels licenses the MSCs in vivo, which would increase their immunosuppressive capacity. Conversely, despite decreasing inflammation, the late administration of MSCs when plasma cytokine levels are low was not as effective in reducing the clinical score of EAE as the early treatment and even induced the appearance of atypical signs of EAE, but no association with increased Th17 cell response was found. Finally, we postulate that the effect of MSCs administration, independent of the time of administration, was able to diminish the lymphocyte infiltration into the brain, probably due to a restoration of the blood-brain barrier (BBB) consequence of the decrease level of IL-6.

\section{Methods}

2.1. Animals. Females C57BL/6 mice, 8-14 weeks old, were purchased from the Central Animal Facility, Faculty of Medicine, University of Chile. Animals were housed under standard laboratory conditions and with food and water ad libitum. Experimental procedures and protocols were performed according to the US National Institutes of Health Guide for the Care and Use of Laboratory Animals (NIH publication number 85-23, revised in 1996) and were approved by the Institutional Animal Care and Use Committee of the Universidad de los Andes and the Fondecyt Bioethics Advisory Committee in Chile.

2.2. MSCs Isolation and Culture. Murine bone marrowderived stem cells (MSCs) were obtained from 8-10-weekold female C57BL/6 mice, as previously described [20]. Bone marrow stromal cells were collected by flushing femurs and tibias with sterile phosphate-buffered saline (PBS) and washing by centrifugation at $350 \times \mathrm{g}$ for $10 \mathrm{~min}$ at room temperature. After centrifugation, cells were plated in $75 \mathrm{~cm}^{2}$ tissueculture flasks at density of $2 \times 10^{6} / \mathrm{cm}^{2}$ in complete alpha Modified Eagle's Medium ( $\alpha$-MEM, Gibco, Auckland, New Zealand), containing $15 \%$ heat-inactivated MSC-qualified fetal bovine serum (FBS, Gibco), $100 \mathrm{U} / \mathrm{mL}$ penicillin, and $100 \mathrm{ug} / \mathrm{mL}$ streptomycin (Gibco) at $37^{\circ} \mathrm{C}$ in a $5 \% \mathrm{CO}_{2}$ atmosphere. Nonadherent cells were removed after $72 \mathrm{hrs}$ and the remaining cells were cultured until reaching $90 \%$ confluence and the medium was replenished every 3 days. After 2 passages, cells were isolated by negative selection using antiCD45 microbeads (Miltenyi, Auburn, CA) according to the manufacturer's protocol. CD45 negative cells were cultured at 10.000 cells $/ \mathrm{cm}^{2}$ in complete $\alpha-M E M$ medium at $37^{\circ} \mathrm{C}$ and 
$5 \% \mathrm{CO}_{2}$. After 2-3 passages, adherent cells were characterized for adipogenic, chondrogenic, and osteogenic differentiation as previously described [20]. Phenotypic characterization for hematopoietic and mesenchymal cell antigens and immunosuppressive capacity was performed by flow cytometry.

2.3. Flow Cytometry Analysis. MSCs phenotype was confirmed by flow cytometry based on the positivity for CD29, CD44, Sca-1, and CD90 and the absence of CD45 and CD34 antigens (all antibodies were purchased from BD Biosciences, conjugated to FITC or PE). Surface staining was performed following standard protocol. The samples were acquired in a Beckman Coulter XL flow cytometer. Data were analyzed using FCS Express 4 Plus Research Edition software.

2.4. Suppression of Splenocyte Proliferation. Splenocytes were isolated from C57BL/6 mice using a 70 um cell strainer in PBS buffer and stained with CFSE according to the manufacturer's instructions. CFSE-splenocytes were cultured in the presence or absence of MSCs in a 1:10 ratio (MSCs : splenocytes) in a complete RPMI medium, $10 \%$ FBS, $2 \mathrm{mM}$ L-glutamine, $50 \mu \mathrm{M} \beta$-mercaptoethanol, $100 \mathrm{U} / \mathrm{mL}$ penicillin, and $100 \mu \mathrm{g} / \mathrm{mL}$ streptomycin (Gibco) at $37^{\circ} \mathrm{C}$ in a $5 \% \mathrm{CO}_{2}$ atmosphere. Cells were stimulated by adding Concanavalin A $(3 \mu \mathrm{g} / \mathrm{mL})$ and cocultures were grown for 5 days. Nonadherent cells were then harvested and surfacestained using PE-Cy5-CD4 antibody. Samples were analyzed by flow cytometry as previously described.

2.5. EAE Induction and MSCs Administration. Female C57BL/6 mice, 10 to 14 weeks old, were injected subcutaneously (s.c.) in the flank with $50 \mu \mathrm{g}$ of $\mathrm{MOG}_{35-55}$ peptide (LifeTein LLC, USA), emulsified in complete Freund's adjuvant (Difco Laboratories, Detroit, MI), and supplemented with heat-inactivated Mycobacterium tuberculosis H37RA (Difco Laboratories, Detroit, MI). Two and fortyeight hours later, mice received intraperitoneally (i.p.) $300 \mathrm{ng}$ of Pertussis toxin (Calbiochem, La Jolla, CA). Clinical signs appeared after 10 days of EAE induction. MSCs $\left(1 \times 10^{6}\right)$ diluted in PBS were intravenously (i.v.) administrated at different stages of the disease, at the onset of the disease (day $10, \mathrm{MSC}_{\text {onset }}$ ), at the peak of the disease (day 18, $\mathrm{MSC}_{\text {peak }}$ ), or at the time of EAE stabilization (day 30, $M C_{\text {late }}$ ) (Figure 2(a)). The clinical scores and mice weights were recorded daily for 50 days. Animals were evaluated according to previously published scoring scales [29]. The classical EAE scores were assigned as follows: score 0 , no disease; score 0.5 , reduced tail tonus; score 1, limp tail; score 1.5, limp tail and ataxia; score 2, limp tail, ataxia, and hind limb weakness; score 2.5 , at least one hind limb paralyzed/weakness; score 3, both hind limbs paralyzed/weakness; score 3.5, complete paralysis of hind limbs; score 4, paralysis until hip; score 5, dead or dying animal. The nonclassical (atypical) EAE scores were assigned as follows: score 0 , no disease; score 1 , head tilted slightly (ataxia, no tail paralysis); score 2, head tilted severely; score 3 , inability to walk on a straight line; score 4 , laying on side; score 4.5, rolling continuously unless being supported; score 5 , dead or dying animal. Representative photographs of classic (Figure 2(b)) and atypical (Figure 2(c)) score are shown. For both scoring scales, the daily mean clinical scores and cumulative scores were calculated.

2.6. ELISA. Plasma concentrations of a broad panel of cytokines were measured by a Milliplex mouse Th17 magnetic bead panel kit (catalogue number MTH17MAG-47K, Millipore). The panel analyzed was IFN- $\gamma$, TNF- $\alpha$, IL- $1 \beta$, IL- 6 , IL4 , IL-10, TNF- $\beta$, IL-17A, IL-17F, and IL-23. Plasma samples were obtained by centrifugation $(300 \times \mathrm{g})$ for $20 \mathrm{~min}$ at different time points after EAE induction for each experimental group. Individual plasma samples of each group at specific time were pooled and analyzed in triplicate according to the manufacturer's instructions on Luminex 200, EMD Millipore Corporation.

2.7. Real Time PCR. Total RNA was extracted from spinal cords and from brain left hemispheres using Trizol reagent (Invitrogen) and treated with DNAse I (Fermentas). Two $\mu \mathrm{g}$ of DNAse I treated RNA was reverse-transcribed using ImProm RT and random hexamers (Promega) in $30 \mu \mathrm{L}$ total volume reaction according to the manufacturer's recommendations. PCR was performed using $2.5 \mu \mathrm{L}$ of diluted cDNA $(1: 100-1: 500)$ and $10 \mu \mathrm{L}$ of primer-containing GoTaq Master Mix (Promega, 150 pmol each primer) and analyzed using Mx3000P qPCR system (Agilent Technologies). Primers used were as follows: $5^{\prime}$ - AGC AGT GTG GAC CGT AGA TGA $3^{\prime}$ (FoxP3, sense), $5^{\prime}$ - GGC AGG GAT TGG AGC ACT T - $3^{\prime}$ (FoxP3, antisense), $5^{\prime}$ - AGT GTA ATG TGG CCT ACT CCT $-3^{\prime}$ (ROR $\gamma$ T sense), $5^{\prime}$ - GGC TGG TTC GGT CAA TGG G - $3^{\prime}$ (ROR $\gamma$ T, antisense), $5^{\prime}$ - AAC CGC TTA TAT GTC CAC CCA $-3^{\prime}$ (T-bet, sense), $5^{\prime}$ - CTT GTT GTT GGT GAG CTT TAG C - $3^{\prime}$ (T-bet, antisense), $5^{\prime}$ - CGG ACA GGA TTG ACA GAT TG $-3^{\prime}(18 S$, sense $)$, and $5^{\prime}$-CAA ATC GCT CCA CCA ACT AA $-3^{\prime}$ (18S, antisense). Expression level of transcripts was normalized to $18 \mathrm{~S}$ mRNA levels (normalizer) and to control healthy mice (control) according to the formula $2^{-\Delta(\Delta \mathrm{CT})}[30]$.

2.8. Statistical Analysis. Statistical analyses were performed using the software GraphPad Prism 5.0 (San Diego, CA, USA). Unpaired Mann-Whitney or Kruskal-Wallis tests were used to compare between the different experimental groups. Data were expressed as mean \pm SEM. All $P$ values $<0.05$ were considered statistically significant.

\section{Results}

3.1. MSCs Characterization. Following cell isolation, adherent cells with fibroblast-like phenotype in passage 3 were characterized following the mesenchymal stem cell minimal criteria [31]. MSCs showed high expression of the classical mesenchymal stem cells surface markers CD44, CD29, and Sca-1, milder expression of CD90 and absence of hematopoietic markers CD34 and CD45 (Figure 1(a)). The plasticity assay demonstrates that MSCs were able to differentiate into osteoblasts, chondrocyte, and adipocytes under the appropriate culture conditions as described by Carrión et al. [20]. The suppressive capacity of MSCs was determined using a proliferation assay with CFSE-splenocytes stimulated with 

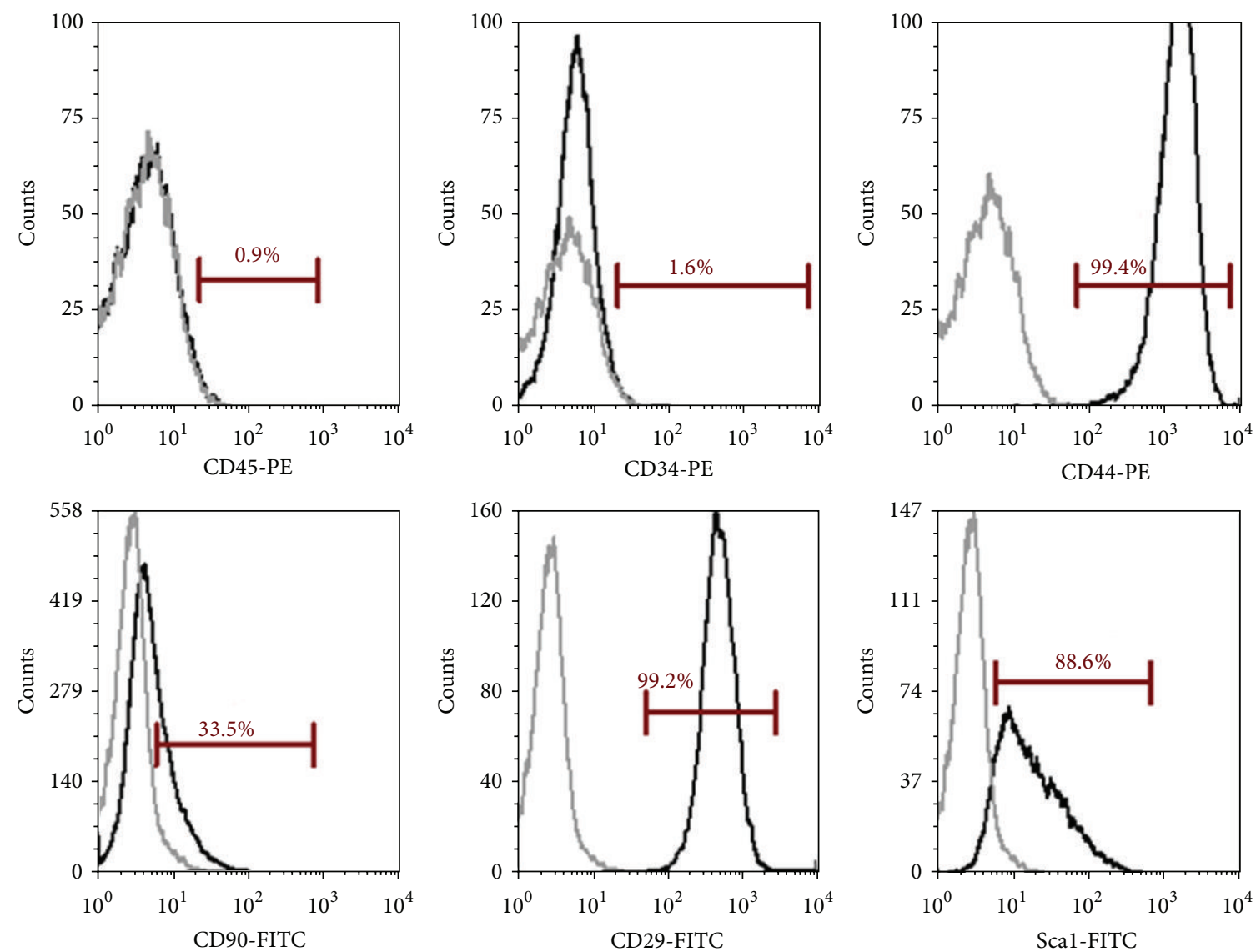

(a)

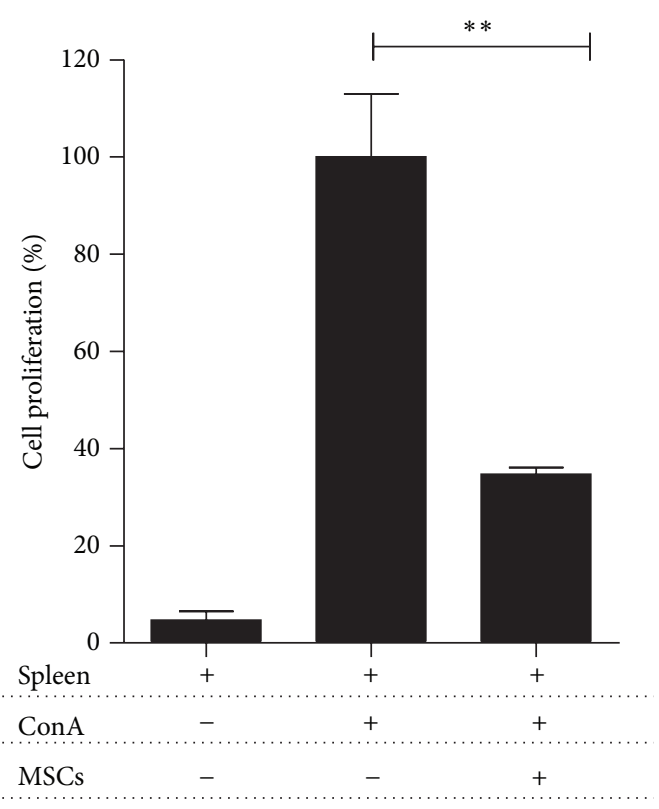

(b)

FIGURE 1: Murine MSCs characterization. (a) Phenotypic characterization of MSCs. Cells were stained for classical murine MSCs markers (CD45, CD34, CD44, CD90, CD29, and Sca-1) and evaluated by flow cytometry using a Beckman Coulter Epics XL and FCS Express V4 Plus Research Edition software. Representative histograms for each antigen are shown in black and overlapping isotype controls in grey. (b) Immunosuppressive capacity of MSCs. Splenocytes were CFSE-stained and stimulated with Concanavalin A ( $3 \mu \mathrm{g} / \mathrm{mL})(\mathrm{ConA})$ in the presence or absence of MSCs for 5 days. Nonstimulated cells were used as negative controls. T cell proliferation was evaluated by flow cytometry, gating on CD4+ cells. Results are shown as the mean \pm SEM. $P$ values were calculated using the unpaired Mann-Whitney test $\left({ }^{* *} P<0.005\right), n=3$. 

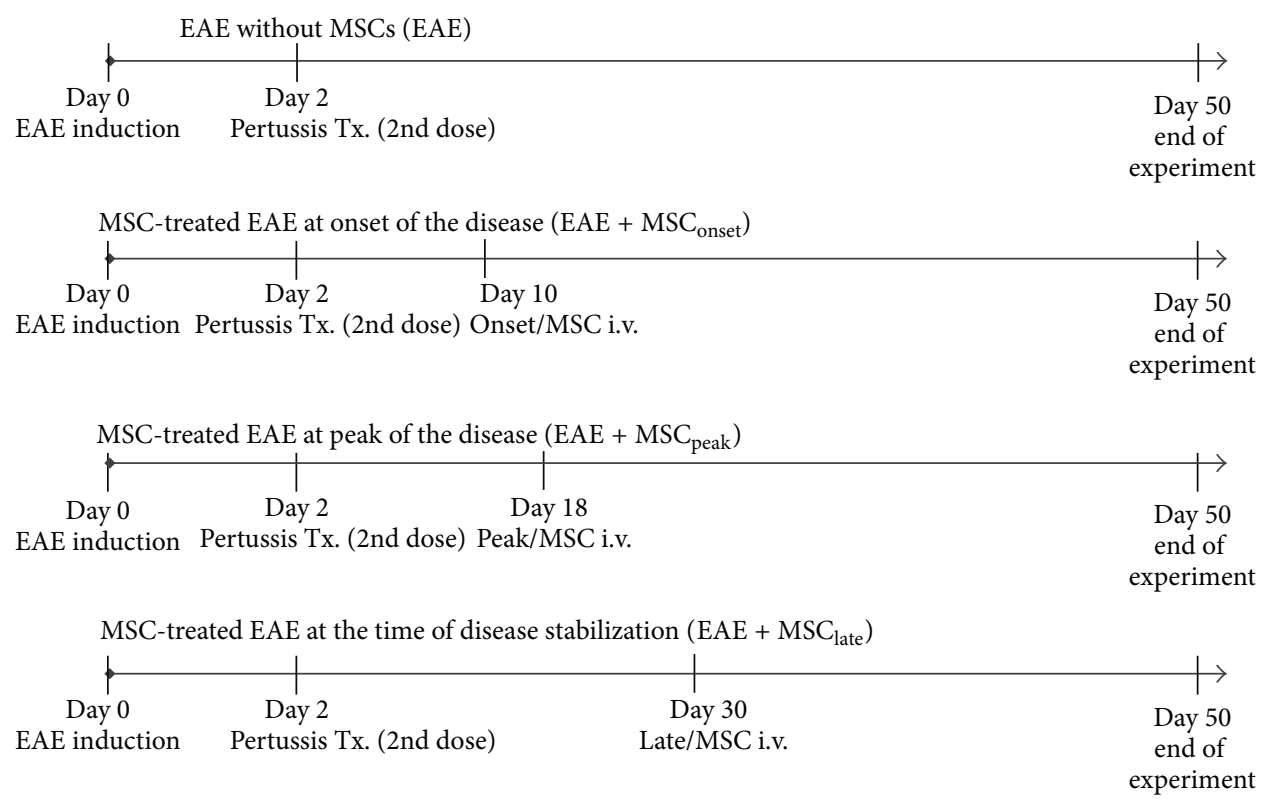

(a)

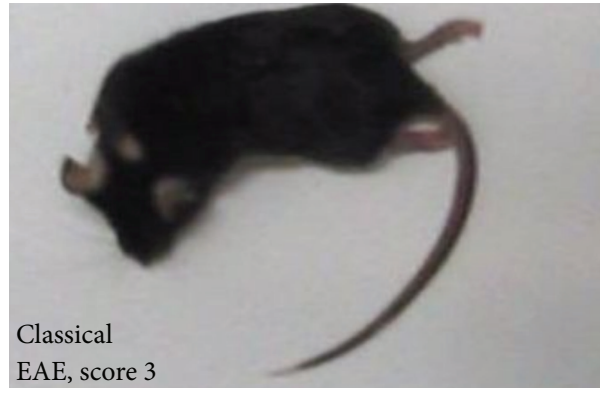

(b)

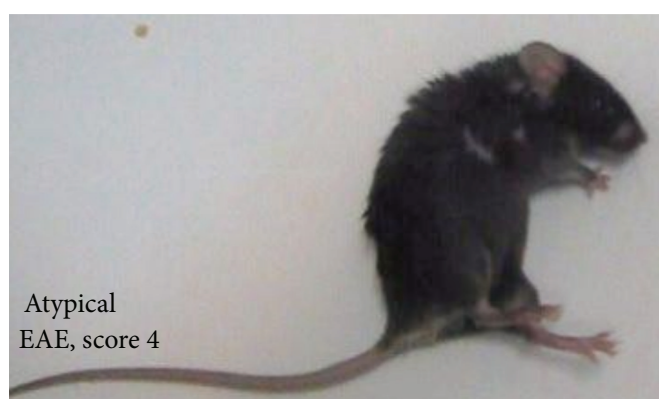

(c)

FIGURE 2: Experimental autoimmune encephalomyelitis model (EAE). (a) EAE induction. C57BL/6 mice were subcutaneously injected with $50 \mu \mathrm{g}$ of $\mathrm{MOG}_{35-55}$, emulsified with CFA and Mycobacterium tuberculosis (4 mg/mL). 2 and $48 \mathrm{hrs} \mathrm{later,} \mathrm{mice} \mathrm{were} \mathrm{intraperitoneally} \mathrm{injected}$ with Pertussis toxin $(350 \mathrm{ng} / \mathrm{mL})$. MSCs $\left(1 \times 10^{6}\right)$ were intravenously administrated either at the onset $\left(\mathrm{EAE}+\mathrm{MSC}_{\text {onset }}\right)$, at peak of the disease $\left(\mathrm{EAE}+\mathrm{MSC}_{\text {peak }}\right)$, or at the time of EAE stabilization $\left(\mathrm{EAE}+\mathrm{MSC}_{\text {late }}\right)$. After EAE induction, animal groups were evaluated daily for both clinical score and animal weight for a total of 50 days. (b-c) Representative photographs of EAE mice manifesting the distinctive classical (b) and atypical (c) signs of the disease and their respective clinical scores are shown.

Concanavalin A (ConA). After 5 days in coculture, MSCs inhibit the proliferation in $60 \%\left({ }^{* *} P<0.005\right)$ (Figure $\left.1(\mathrm{~b})\right)$.

\subsection{MSCs Ameliorate Classical Clinical Symptoms in EAE} Animals. To elucidate the clinical effect of MSCs administration at different time points of EAE progression, MSCs were injected $\left(1 \times 10^{6}\right.$ cells/mice $)$ at the onset of the disease (day $\left.+10, \mathrm{MSC}_{\text {onset }}\right)$, at the peak of the disease $\left(\right.$ day $\left.+18, \mathrm{MSC}_{\text {peak }}\right)$, or at the time of EAE stabilization (day $+30, \mathrm{MSC}_{\text {late }}$ ) (Figure 2(a)). The classical and atypical clinical scores were evaluated for 50 days. Consistent with previous reports [14], MSCs injected at the onset of the disease induced a significant improvement of classical clinical score in EAE animals $\left({ }^{*} \mathrm{P}<\right.$ 0.05) (Figure 3(a)). Moreover, a significant reduction of the area under curve (AUC) was detected after one (Days 11-

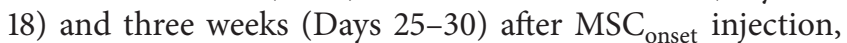
compared to the EAE group without MSCs $\left({ }^{*} P<0.05\right)$
(Figure 3(b)). MSCs administered at the peak of the disease induced a robust but transient improvement of clinical signs of EAE $\left({ }^{*} P<0.05\right.$ ) (Figure 3(c)). Despite this transient effect, a significant AUC was detected after one (Days 19-24) and even two weeks (Days 25-30) after $\mathrm{MSC}_{\text {peak }}$ injection $\left({ }^{*} \mathrm{P}<\right.$ 0.05) (Figure 3(d)). Later administration of MSCs, at day 30, had no effect on clinical signs of classical EAE (Figure 3(e)), and we did not find differences in AUC analysis (Figure 3(f)).

3.3. MSCs Induced Atypical Signs of EAE. Atypical EAE is characterized by unbalanced gaits or rotatory defects. The atypical condition was followed up by specific clinical score as was previously described by Domingues et al. [29]. We observed that MSCs injections were able to induce the appearance of EAE atypical signs (Figure 2(c)). An incidence of atypical EAE of $66 \%$ and $55 \%$ was observed in those animals injected at the peak $\left(\mathrm{EAE}+\mathrm{MSC}_{\text {peak }}\right)$ and at the time 

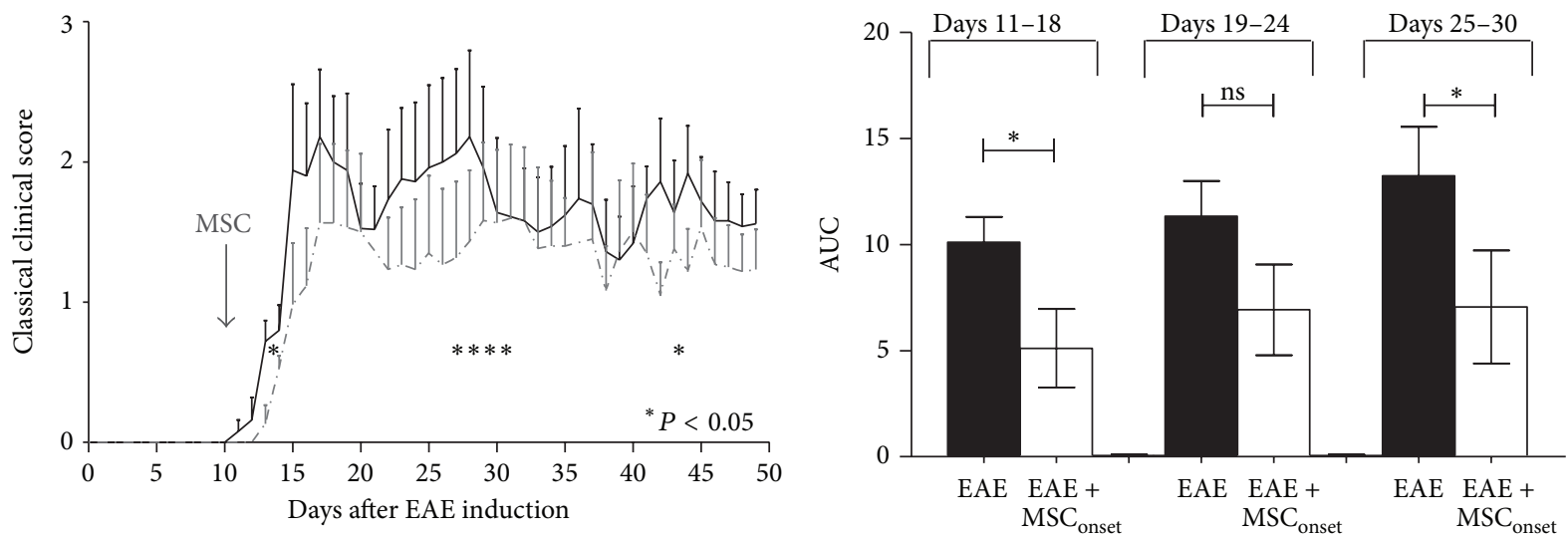

- EAE

... $\mathrm{EAE}+\mathrm{MSC}_{\text {onset }}$

(a)

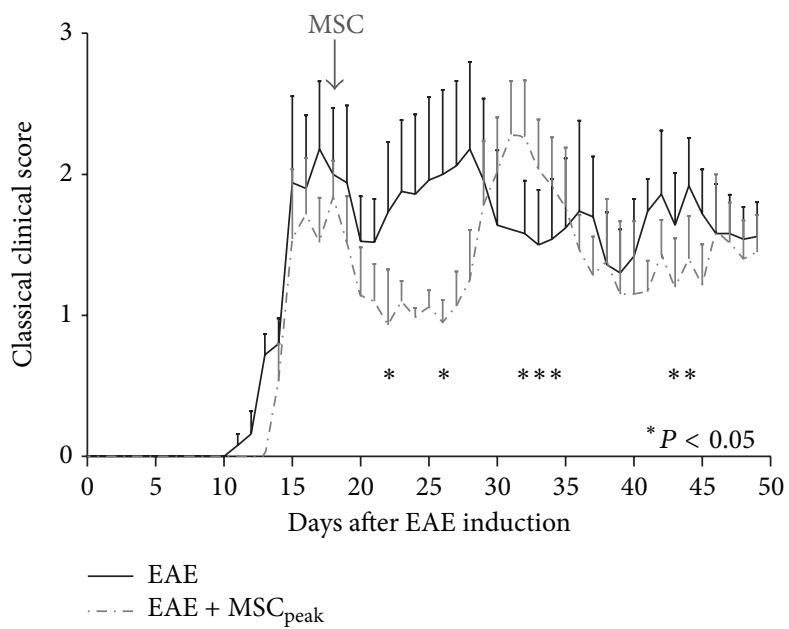

(c)

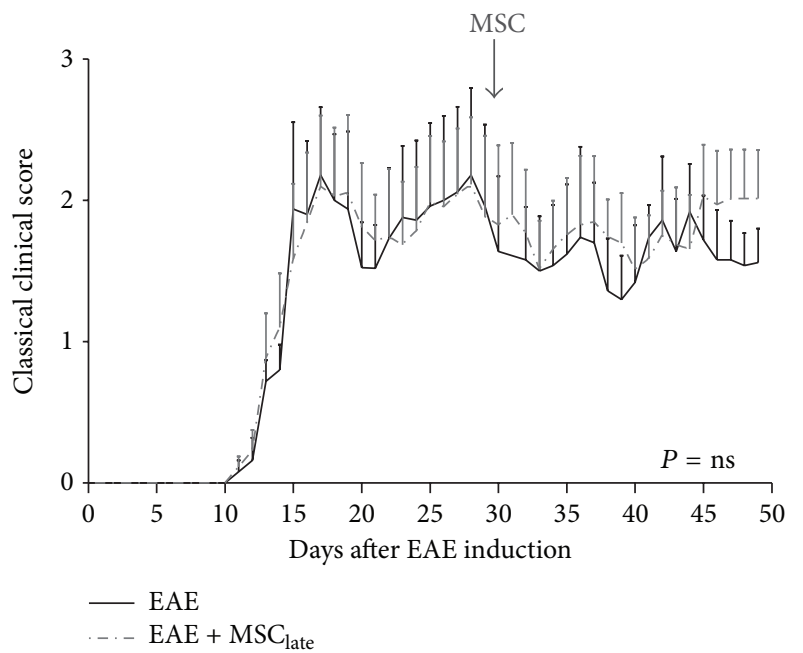

(e) (b)

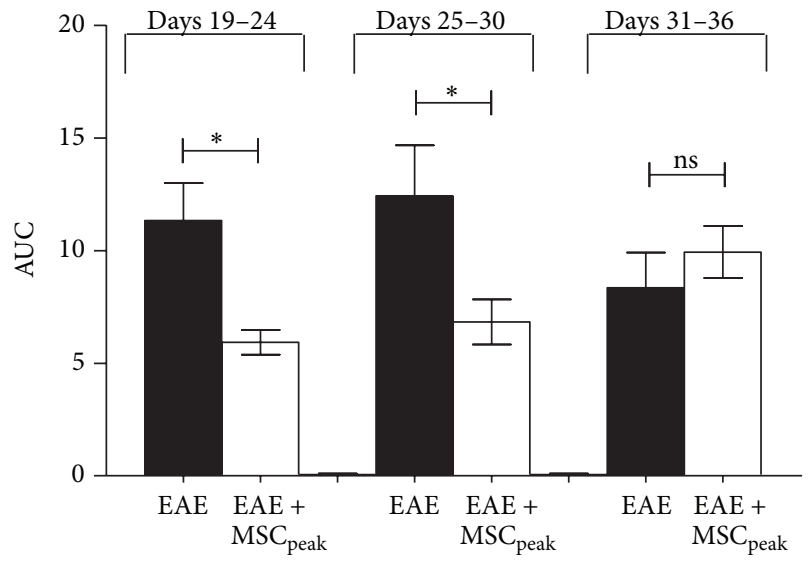

(d)

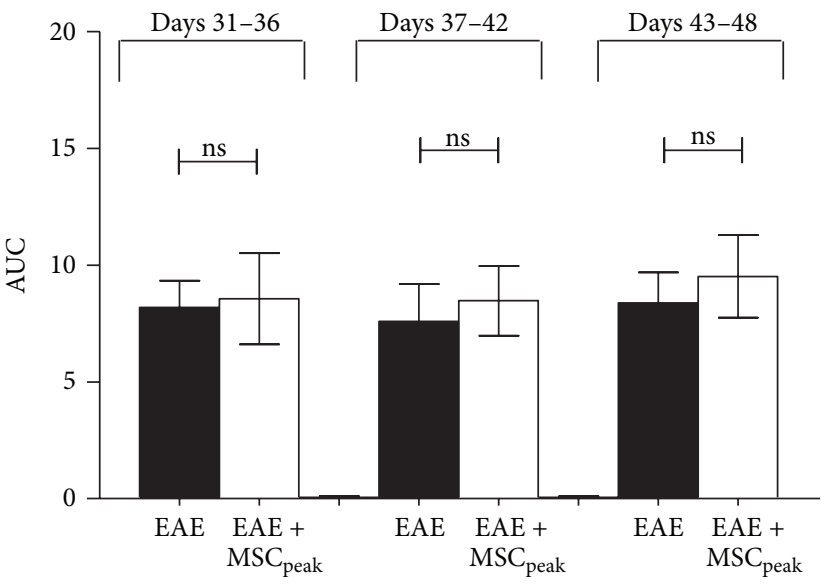

FIGURE 3: MSCs injections at the onset and at the peak of disease induce an improvement of classical EAE. C57BL/6 mice were immunized with $\mathrm{MOG}_{35-55}$ to induce EAE and classical score was evaluated daily. MSCs $\left(1 \times 10^{6}\right)$ were intravenously injected either at $(\mathrm{a}, \mathrm{b})$ the onset of EAE symptoms $\left(E A E+M_{\text {onset }}\right),(c, d)$ at the peak of the disease $\left(E A E+M_{\text {peak }}\right)$, or $(e, f)$ at the time of EAE stabilization $\left(E A E+M_{\text {late }}\right)$. Daily clinical score (a, c, e) and the area under curve (AUC) (b, d, f) parameters were evaluated after MSCs injection as described in Methods and AUC were plotted at specific time periods as indicated. Line curves and bars represent the mean \pm SEM; statistical differences were calculated using Mann-Whitney test $\left({ }^{*} P<0.05\right), n=7$. 


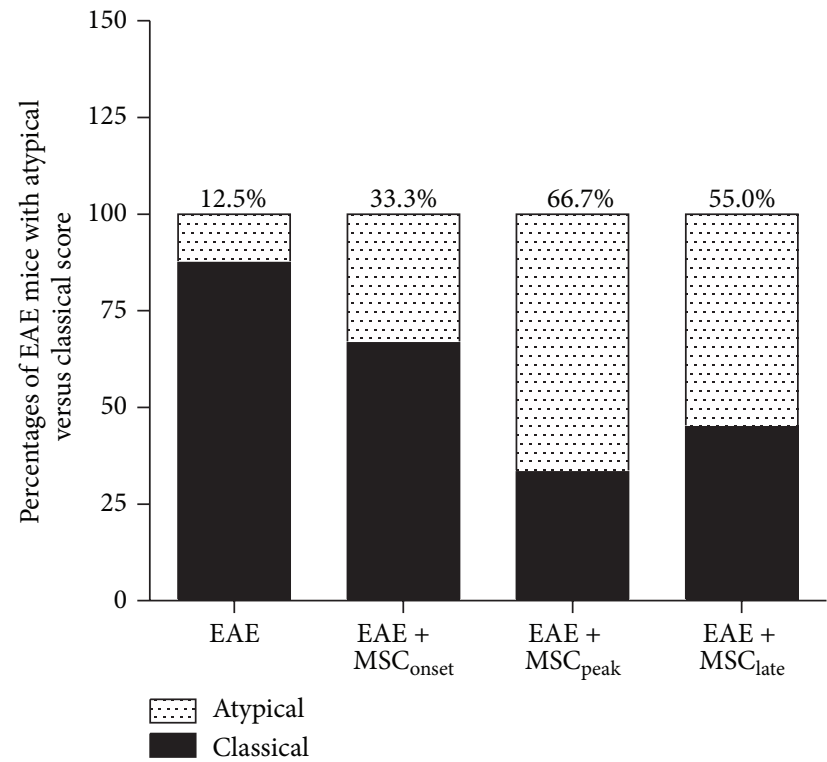

(a)

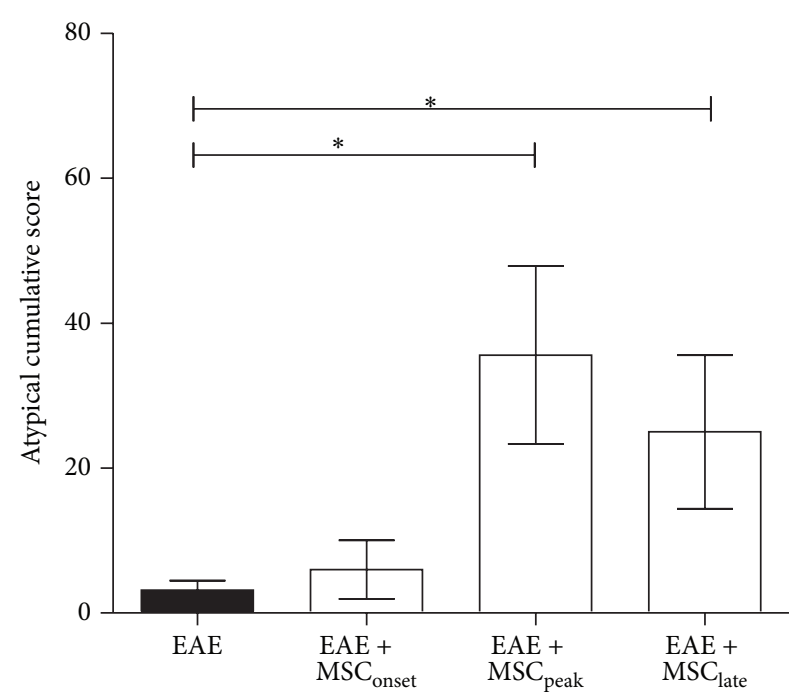

(b)

FIGURE 4: MSCs injections at the peak of EAE and at the time of EAE stabilization induce the appearance of atypical signs. EAE was induced on C57BL/6 mice by $\mathrm{MOG}_{35-55}$ immunization and both classical and nonclassical atypical scores were evaluated as described in Methods. (a) Incidence of classical versus atypical EAE was calculated as the percentage of EAE mice with atypical signs over EAE mice with classical phenotype at the end of the experiment. (b) Atypical cumulative scores were calculated for untreated EAE mice (EAE) and for EAE mice treated with MSCs at the onset $\left(\mathrm{EAE}+\mathrm{MSC}_{\text {onset }}\right)$, at the peak $\left(\mathrm{EAE}+\mathrm{MSC}_{\text {peak }}\right)$, and at the time of EAE stabilization $\left(\mathrm{EAE}+\mathrm{MSC}_{\text {late }}\right)$. Bars represent the mean \pm SEM. Statistical differences were calculated using Mann-Whitney test $\left({ }^{*} P<0.05\right), n=7$.

of EAE stabilization (EAE $\left.+\mathrm{MSC}_{\text {late }}\right)$, respectively. These percentages were much higher than in EAE mice without MSCs treatment (EAE, 12.5\%) or in EAE mice injected at the onset with MSCs (EAE + MSC $_{\text {onset }}, 33.3 \%$ ) (Figure 4(a)). This phenomenon was better detected, when the atypical cumulative score was compared with EAE mice treated with MSCs versus those untreated (Figure 4(b)). Higher atypical cumulative scores were observed in EAE mice treated with MSCs at the peak of the disease $\left(\mathrm{EAE}+\mathrm{MSC}_{\text {peak }}\right)$ and at the time of EAE stabilization $\left({ }^{*} P<0.05\right.$ ) (Figure $\left.4(\mathrm{~b})\right)$. We did not observe an important appearance of atypical signs when MSCs were administered at the onset or to untreated animals (EAE). These results suggest that late MSCs treatments may predispose to the appearance of atypical EAE, and therefore we wanted to analyze CNS infiltration of different types of $\mathrm{T}$ helper lymphocytes that are associated with these clinical signs using a molecular biology approach.

3.4. MSCs Diminished the Presence of T-bet, ROR $\gamma$ T, and Foxp3 in Brains of EAE Mice. The presence of different subsets of $\mathrm{T}$ helper lymphocytes was analyzed in the CNS of these animals by qRT-PCR targeted to specific transcription factors: T-bet, $\operatorname{ROR} \gamma \mathrm{T}$, and Foxp3 associated with Th1, Th17, and Treg, respectively. Evaluation of Th1, Th17, and Treg by qRT-PCR was validated by comparative analysis between qRT-PCR and flow cytometry using in vitro differentiated Th1, Th17, Treg, and control cells (Supplementary Methods and Supplementary Figure 1 available online at http://dx.doi.org/10.1155/2015/140170). Fifty days after EAE induction, animals were sacrificed, brain and spinal cord samples were obtained and then total RNA was isolated as described on methods. A significant increase was detected in T-bet $\left({ }^{* *} P<0.005\right), \operatorname{ROR} \gamma \mathrm{T}\left({ }^{*} P<0.05\right)$, and Foxp3 $\left({ }^{*} P<0.05\right)$ in brains samples on EAE group at day 50 after EAE induction (Figure 5). The treatment with MSCs, independently of the time of administration, significantly decreased the expression level of T-bet, ROR $\gamma \mathrm{T}$, and Foxp3, suggesting a lower presence of Th1, Th17, and Treg lymphocytes $\left({ }^{*} P<0.005,{ }^{* *} P<0.005\right.$ as indicated in Figures 5(a), 5(b), and 5(c)). The expression of T-bet, ROR $\gamma \mathrm{T}$, and Foxp3 in MSCs treated groups was similar to the expression level of healthy mice, and significant differences were not found between MSCs treated groups. A slight increase of Tbet/ROR $\gamma \mathrm{T}$ ratio was observed in $\mathrm{EAE}+\mathrm{MSC}_{\text {late }}$ and $\mathrm{EAE}$ group untreated with MSCs, suggesting a predominance of Th1 lymphocytes in those groups which showed the highest classical clinical score (Figure 5(d)).

Analysis of Foxp3 expression in different MSCs treated groups showed an important decrease of Foxp3 in EAE + $\mathrm{MSC}_{\text {peak }}$ and EAE $+\mathrm{MSC}_{\text {late }}\left({ }^{* *} P<0.005\right)$ but it was not so significant in $\mathrm{EAE}+\mathrm{MSC}_{\text {onset }}$ (Figure $5(\mathrm{c})$ ). In addition, highest Foxp3/T-bet and Foxp3/ROR $\gamma \mathrm{T}$ ratios were detected in brain samples of EAE $+\mathrm{MSC}_{\text {onset }}\left({ }^{*} P<0.005,{ }^{* *} P<\right.$ 0.005 as indicated in Figures 5(e) and 5(f)), suggesting a predominance of Treg over Th1 and Th17 lymphocytes. The Foxp3/ROR $\gamma$ T and Foxp3/T-bet ratios were $<1$ when MSCs 


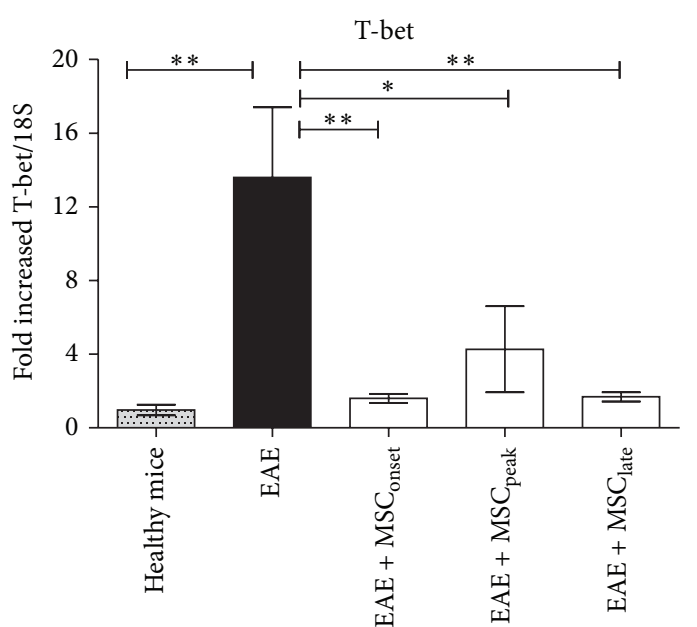

(a)

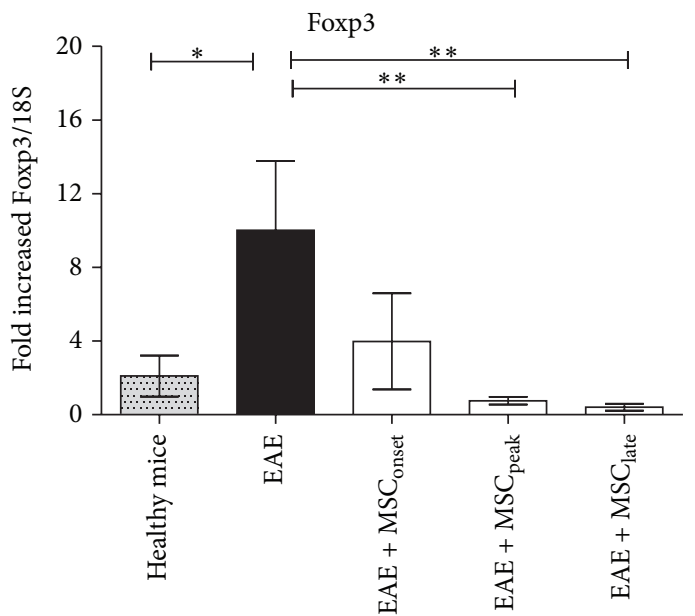

(c)

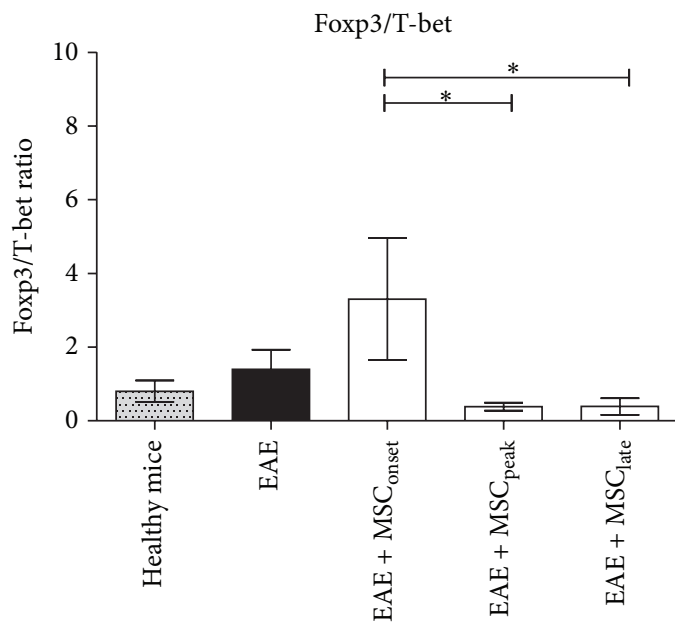

(e)

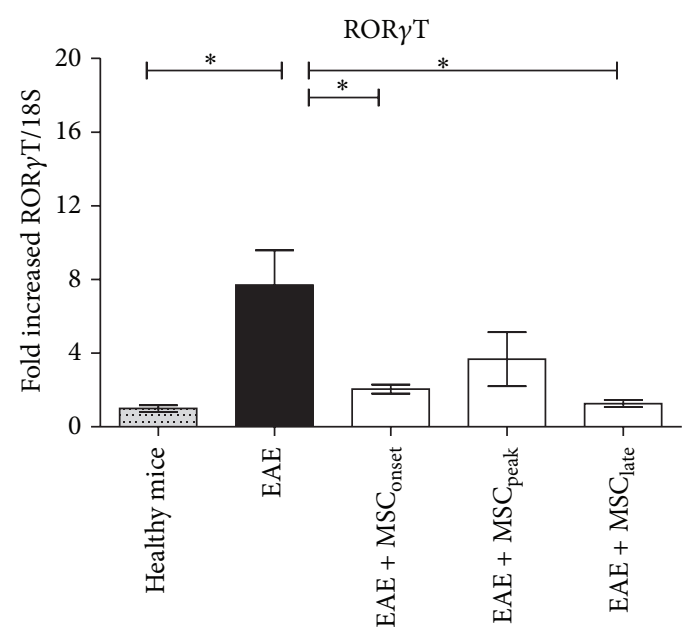

(b)

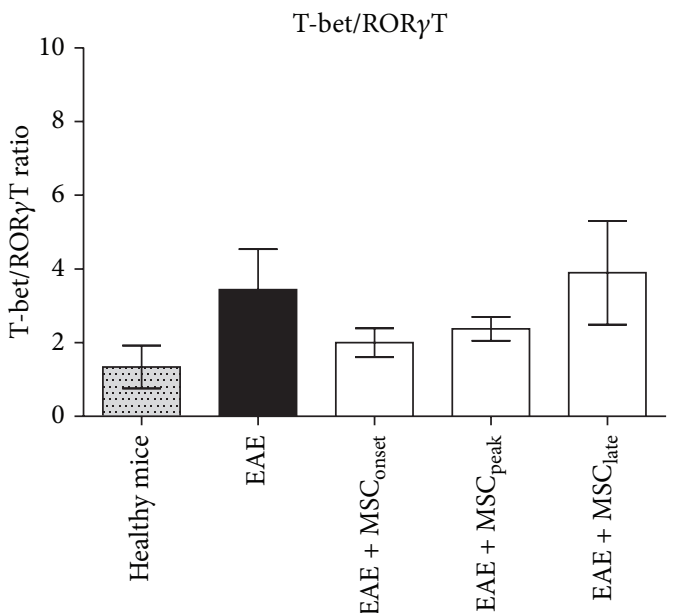

(d)

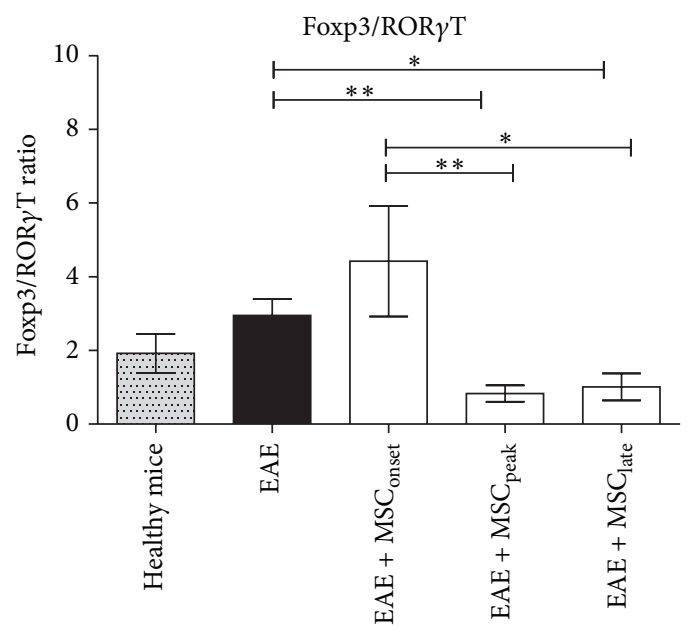

(f)

FIGURE 5: MSCs injections at different stages of EAE diminished brain T-bet, ROR $\gamma$ T, and Foxp3 mRNA levels. Healthy C57BL/6, EAE mice, or EAE mice injected with MSCs $\left(1 \times 10^{6}\right)$ at the onset $\left(\mathrm{EAE}+\mathrm{MSC}_{\text {onset }}\right)$, at the peak $\left(\mathrm{EAE}+\mathrm{MSC}_{\text {peak }}\right)$, and at the time of EAE stabilization $\left(\mathrm{EAE}+\mathrm{MSC}_{\text {late }}\right.$ ) were sacrificed after 50 days of EAE progression. Total brain RNA was extracted (Trizol) and $2 \mu \mathrm{g}$ of DNAse-treated RNA was retrotranscribed as described in Methods. Relative mRNA levels of bona fide transcriptions factors associated with CD4+ T helper subtypes Th1 (T-bet, (a)), Thl7 (ROR $\gamma T$, (b)), and Treg (Foxp3, (c)) were determined by RT-qPCR using the $2^{-\Delta \Delta C t}$ method. Individual ratios, (d) Tbet/ROR $\gamma$ T, (e) Foxp3/T-bet, and (f) Foxp3/ROR $\gamma$ T were also calculated. Bars in the plots represent the mean \pm SEM. Statistical differences were calculated using Mann-Whitney test $\left({ }^{*} P<0.05,{ }^{* *} P<0.005\right), n=7$. 


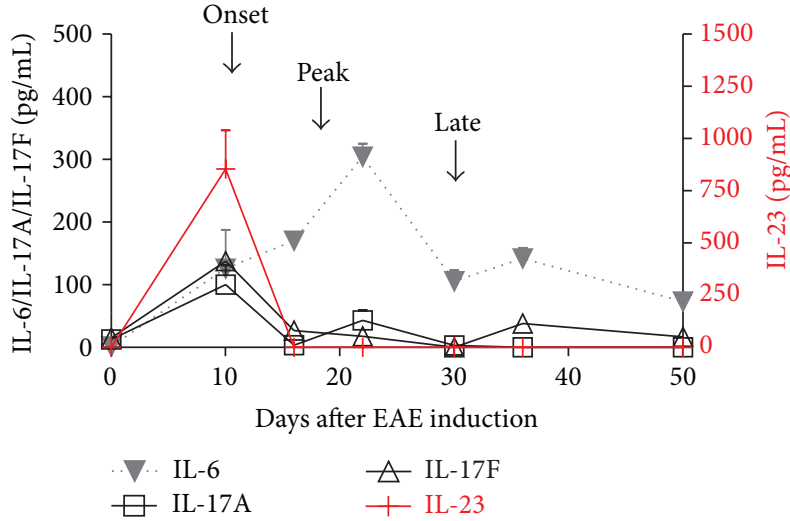

(a)

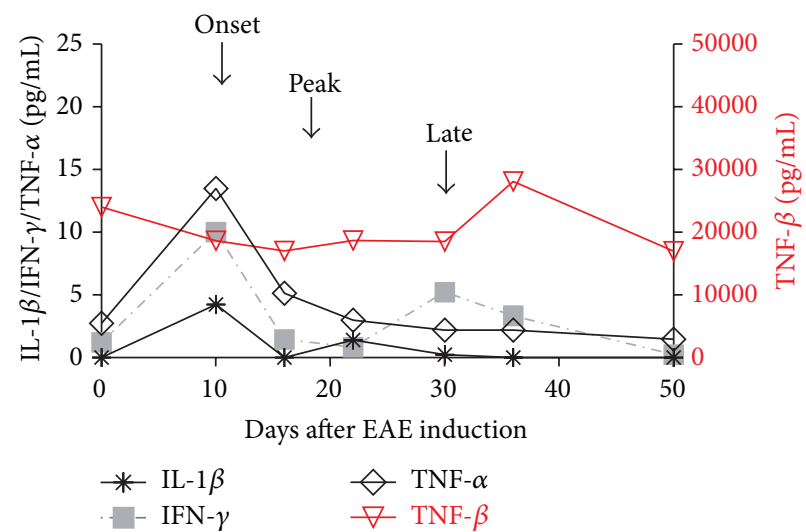

(b)

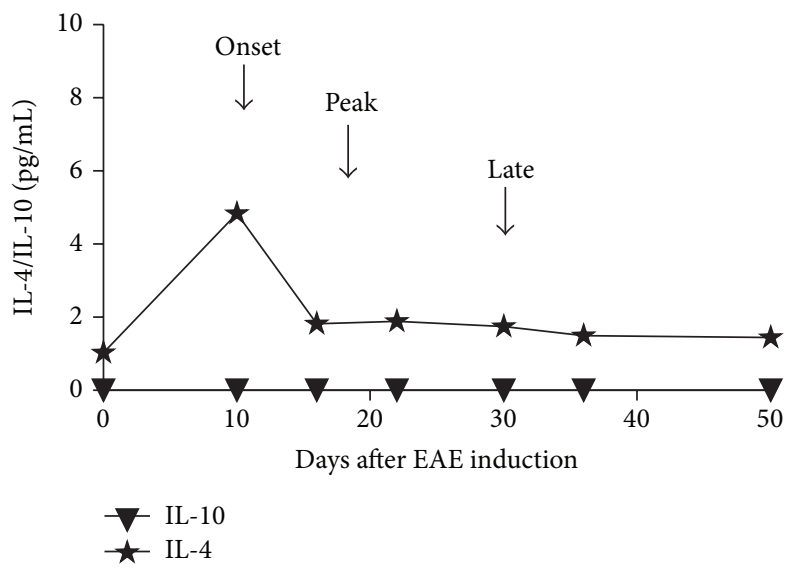

(c)

FIGURE 6: Cytokines levels in plasma samples of EAE mice at the different times of EAE progression. Blood samples were taken at different times of EAE progression, before EAE induction (day 0$)$, at the onset $($ day +10$)$, before the peak $($ day +16$)$, after the peak $($ day +22$)$, at the time of EAE stabilization $($ day +30$)$, after EAE stabilization $($ day +36$)$, and at the end of experiment $($ day +50$)$. Plasma samples were isolated by centrifugation and stored at $-80^{\circ} \mathrm{C}$ until being used. Cytokines levels were detected using Milliplex mouse Th17 magnetic bead panel on Luminex 200 instrument (Merck, Millipore) and the kinetics were plotted of (a) Th1, (b) Th17 cytokines profiles and (c) IL-4 and IL-10 of EAE mice ( $n=7$, each experiment). The samples of each experiment were pooled and analyzed independently in triplicate.

were injected later $\left(\mathrm{EAE}+\mathrm{MSC}_{\text {peak }}\right.$ and $\left.\mathrm{EAE}+\mathrm{MSC}_{\text {late }}\right)$, even lower than healthy mice (Figures 5(e) and 5(f)), suggesting a predominance of proinflammatory $\mathrm{T}$ cells versus Treg in these groups.

Except for a small detection of ROR $\gamma$ T expression in EAE mice treated with MSCs at the peak of the disease, we were unable to detect the expression of T-bet, ROR $\gamma \mathrm{T}$, or Foxp 3 by qRT-PCR in spinal cord samples from all EAE groups at the end of the experiment (data not shown).

\subsection{Evaluation of Inflammatory Profiles at Different Stages} of EAE Progression. In order to evaluate the kinetics of the inflammatory response during EAE progression, the cytokine profile in plasma samples of EAE mice at different time points was assessed. Blood samples were collected either before EAE induction (day 0 ), at the onset $($ day +10$)$, before the peak (day $+16)$, after the peak $($ day +22$)$, at the time of EAE stabilization $($ day +30$)$, after EAE stabilization $($ day +36$)$, or at the end of experiment $(\mathrm{day}+50)$. Ten different cytokines were measured using Milliplex mouse magnetic bead panel kit (IL-6, IL-1 $\beta$, IFN- $\gamma$, TNF- $\alpha$, TNF- $\beta$, IL-23, IL-17 A, IL-17F, IL-4, and IL-10). At the onset of the disease, high levels IL- 6 were detected, as well as cytokines associated with Th1 (IL-1 $\beta$, IFN- $\gamma$, TNF- $\alpha$, and TNF- $\beta$ ) (Figure 6(a)) and Th17 (IL-23, IL-17A, and IL17F) lymphocytes (Figure 6(b)). IL-10 was not detected at any of the time points analyzed and only very low amounts of IL-4 during EAE progression were found (Figure 6(c)). In a separate experiment, IL-27 and IL-17E were also measured and at the onset of EAE high levels of IL-27 and IL-17E were found (Supplementary Figure 2). The inflammatory scenario at the peak of EAE was completely different; at this time the majority of the cytokines had already decreased to levels close to those observed in healthy mice, and only IL-6 plasma level and to a lesser extent TNF- $\alpha$ remained elevated. At the time of EAE stabilization, IL- 6 levels had decreased close to those detected at the onset of EAE, and a slight increase of IFN$\gamma$ in respect to the peak point was observed. Levels of other 


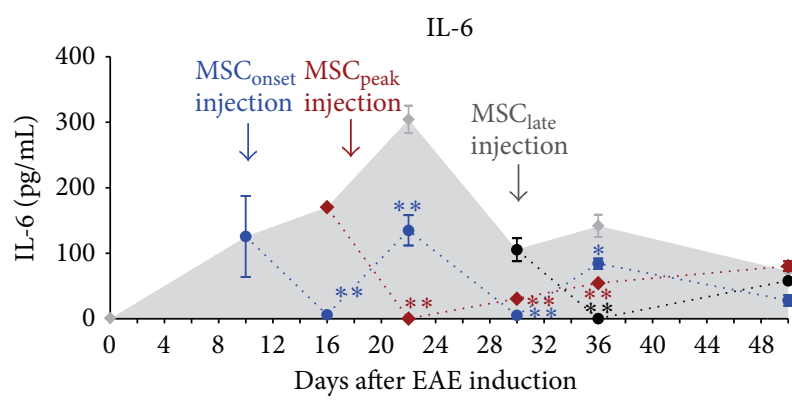

(a)

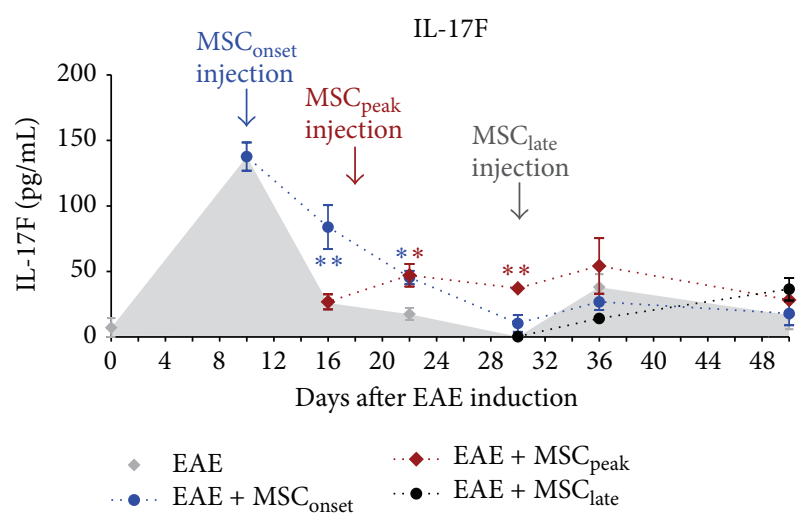

(c)

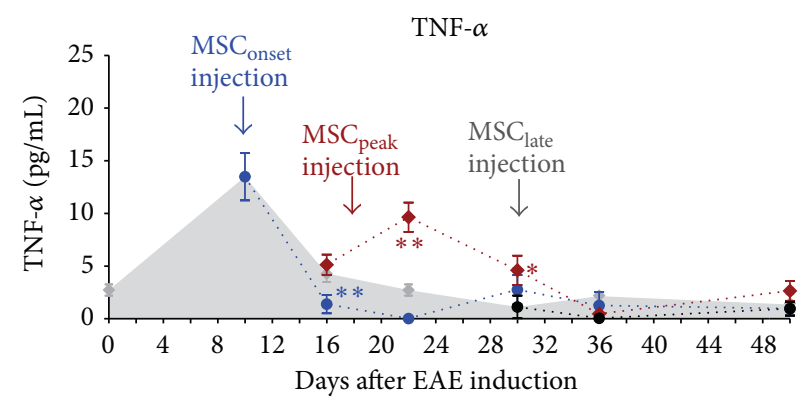

(b)

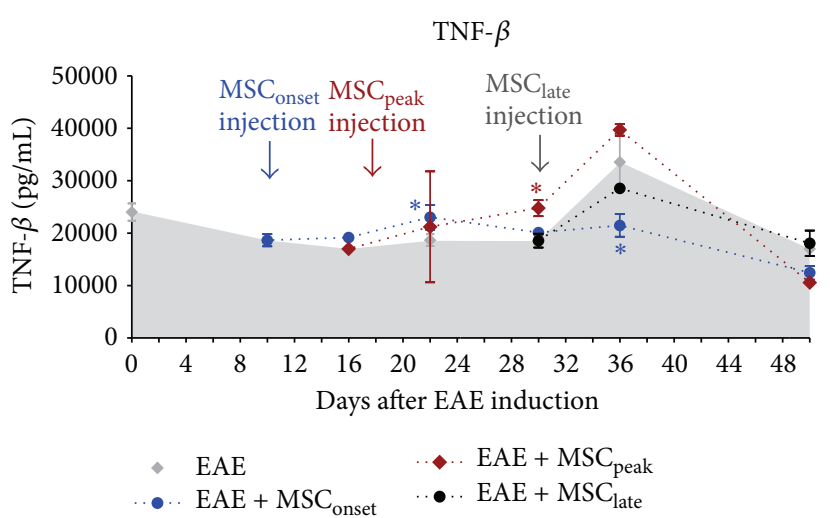

(d)

FIGURE 7: MSCs administration significantly diminished IL-6 plasma levels independently of the time of injection. Plasma samples were isolated from EAE mice, treated or not (EAE) with MSCs at different time of EAE progression, at the onset $\left(\mathrm{EAE}+\mathrm{MSC}_{\text {onset }}\right)$, at the peak $\left(\mathrm{EAE}+\mathrm{MSC}_{\text {peak }}\right)$, and at the time of EAE stabilization $\left(\mathrm{EAE}+\mathrm{MSC}_{\text {late }}\right)$. Blood samples were taken (day 0), at the onset $($ day +10$)$, before the peak $($ day +16$)$, after the peak $($ day +22$)$, at the time of EAE stabilization $($ day +30$)$, after EAE stabilization $($ day +36$)$, and at the end of experiment $($ day +50$)$. Plasma samples were isolated by centrifugation and stored at $-80^{\circ} \mathrm{C}$ until being used. Cytokine levels IL-6 (a), TNF- $\alpha$ (b), IL-17F (c), and TNF- $\beta$ (d) were detected using Milliplex mouse Th17 magnetic bead panel on Luminex 200 (Merck, Millipore). The samples of each EAE group $(n=7)$ were pooled and analyzed in triplicate. Bars represent the mean \pm SEM; statistical differences were calculated using Mann-Whitney test $\left({ }^{*} P<0.05\right)$.

cytokines remained close to those observed in healthy mice (Figure 6).

3.6. MSCs Decreased IL-6 Levels Independently of the Time of MSCs Administration. In order to evaluate the effect of MSCs injections on cytokine plasma levels in EAE mice, we used a Milliplex mouse magnetic bead panel kit to measure Th1, Th17, and anti-inflammatory cytokines as previously described. We observed that MSCs, independently of the time of injection, significantly decreased IL-6 levels and this effect was maintained over time $\left({ }^{*} P<0.005,{ }^{* *} P<0.005\right.$ as indicated in Figure 7(a)). MSCs injected at the onset of EAE were also able to decrease the levels of TNF- $\alpha\left({ }^{* *} P<0.005\right)$ (Figure $7(\mathrm{~b}))$. We also observed an increase of IL-17F $\left({ }^{* *} P<\right.$ $0.005)$ (Figure $7(\mathrm{c}))$ and TNF- $\beta\left({ }^{*} P<0.05\right)$ (Figure $7(\mathrm{~d})$ ), without a change in IL-17A levels (Supplementary Figure 3). MSCs injected at the peak of EAE also induced an increase in IL-17F and TNF- $\beta$ levels $\left({ }^{* *} P<0.005,{ }^{*} P<0.05\right.$, resp.) (Figures $7(\mathrm{c})$ and $7(\mathrm{~d})$ ), accompanied also with an increase of TNF- $\alpha\left({ }^{*} P<0.05\right)$ (Figure 7(b)). IL-17A levels were below the limit of detection in this case. Late administration of MSCs in EAE mice, at the time of EAE stabilization, had not a significant effect in any of the cytokines evaluated. However, it is important to mention that cytokine levels were extremely low at this point. Finally, very low levels of IL-4, IL-23, and IFN- $\gamma$ were detected in all EAE groups after the onset of EAE, and no significant changes were observed after MSC administration (Supplementary Figure 3). We did not detect significant plasma levels of IL-10 and IL- $1 \beta$ in any group (data not shown).

\section{Discussion}

In this study, the therapeutic effects of MSCs administration at different times of EAE progression, associated with different inflammatory states of the disease, were evaluated. Unexpectedly, the appearance of atypical signs of EAE was observed when MSCs were injected after the onset of EAE. Atypical EAE is characterized by ataxia and an immune response biased to Th17 phenotype, in striking contrast to classical EAE, characterized by ascending paralysis and Th1 mediated immune responses. The effects of MSCs treatment at different time points during EAE were analyzed to bring awareness of how important is the currently inflammatory condition of the patient, at the moment of MSCs administration as cellular therapy to MS patients. 
It was observed that injections of MSCs at early stages of EAE progression $\left(\mathrm{EAE}+\mathrm{MSC}_{\text {onset }}\right.$ or $\mathrm{EAE}+\mathrm{MSC}_{\text {peak }}$ groups) induced an improvement of daily classical clinical score and also a reduction of the AUC, but no beneficial effect was observed when MSCs were injected at the time of EAE stabilization $\left(\mathrm{EAE}+\mathrm{MSC}_{\text {late }}\right.$ ) (Figure 3). These results correlate with higher levels of proinflammatory cytokines in EAE mice at the time of MSCs administration (Figure 6). These results are in agreement with previously published data showing that early administration of MSCs reduced the disease severity, but no effect was observed when MSCs were injected later [14].

The appearance of atypical signs in EAE mice after MSCs injection is an intriguing and novel finding. It was observed that MSCs significantly induced the appearance of atypical signs of EAE when injected at the peak and at the time of EAE stabilization, but not during early time points (Figure 4). It is presumed that these atypical signs are due to a predominance of Th17 lymphocytes within the CNS $[28,29]$, contrary to classic EAE, which is governed by Th1 lymphocytes [25]. Therefore, the presence of Th1, Th17, and also Treg lymphocytes was evaluated within the CNS after MSCs injections. We observed, independently of the time of administration, that MSCs are able to diminish the expression of T-bet, ROR $\gamma \mathrm{T}$, and Foxp3, suggesting a lesser presence of Th1, Th17, and Treg, respectively, in brain samples (Figure 5). However, decreases of brain proinflammatory lymphocytes were not always associated with an attenuation of the clinical score, especially in EAE $+\mathrm{MSC}_{\text {late }}$ (Figure 3(c)). We speculate that as the damage caused by demyelination in the CNS occurs early, therefore the symptoms might be reversed only by MSCs acting at early time points. Later administration of MSCs, despite decreasing the presence of T cells on EAEbrain, was not efficient for improving clinical signs of EAE.

Although late administration of MSCs induces atypical signs of EAE, no association was found with ROR $\gamma \mathrm{T} / \mathrm{T}$ bet ratio, as previously described by Stromnes et al. These authors evaluated Th1/Th17 ratio by ELISPOT assays using lymphocytes derived of spleen and CNS samples obtained at the peak of EAE [28]. Furthermore, there was no increase of ROR $\gamma \mathrm{t}$ in brain samples (Figure 5(b)) or percentage of $\mathrm{CD}^{+} \mathrm{IL} 7^{+}$cells in lymph nodes as previously described [32], probably because the $\mathrm{T}$ cell analysis was performed fifty days after EAE induction in order to detect late changes in the clinical score. It is expected that, at day 50 after EAE induction, brain inflammation was already resolved and lymphocyte levels are much lower to find differences in Th17 and Th1.

The highest Foxp3/ROR $\gamma \mathrm{T}$ and Foxp3/T-bet ratios were observed in brain samples of EAE mice treated at the onset, suggesting a predominance of Treg over Th1 and Th17. It is possible that the lowest clinical score observed in EAE + $\mathrm{MSC}_{\text {onset }}$ animals may be due to the higher presence of Foxp3 expression (Figure 3(a)). On the contrary, EAE + $\mathrm{MSC}_{\text {peak }}$ and $\mathrm{EAE}+\mathrm{MSC}_{\text {late }}$ showed Foxp3/ROR $\gamma \mathrm{T}$ and Foxp3/T-bet ratios $<1$. These experimental groups also showed a higher incidence of atypical signs. This result suggests that MSCs injection in the most chronic inflammatory microenvironments (i.e., the peak and the stabilization of the disease) may generate an imbalance between Treg cells and inflammatory $\mathrm{T}$ cells, Thl, and Th17 and possibly generate a phenotype defined as atypical EAE. Further experiments including in situ FACS are needed to confirm this.

Previously we described that MSC injected at the peak of EAE significantly reduced the percentage of Th17 cells, increased the percentage of Th1 cells, and increased the percentage of Treg cells on lymph nodes [32]. Now, different results were found in brain samples. Whether this is due to the tissue where the lymphocyte populations are being measured (lymph nodes versus brain tissue) or the method of analysis (flow cytometry versus transcription factor mRNA) was discarded. We performed in vitro differentiation assays using C57BL/6 naïve CD4+ T cells using specific cytokines (see Supplementary Methods) and determined the percentage of $\mathrm{CD} 4^{+} \mathrm{IFN} \gamma^{+}$(Th1), $\mathrm{CD} 4^{+} \mathrm{IL}^{+}{ }^{+}$(Th17), and $\mathrm{CD} 4^{+} \mathrm{Foxp}^{+}$ (Treg) lymphocytes by flow cytometry, as well as the relative expression of T-bet, ROR $\gamma \mathrm{T}$, and FoxP3 by real time PCR in sister cultures for each polarizing condition. A positive correlation between mRNA levels and the amount of cells was observed for specific $\mathrm{T}$ subsets measurements using these two techniques (Supplementary Figures 1a and 1b). Increased T-bet mRNA levels were detected where increased percentage of $\mathrm{CD} 4^{+} \mathrm{IFN} \gamma^{+}$lymphocytes was measured by FACS, indicating that T-bet mRNA levels could be used as an alternate parameter for Thl determination in a sample containing mixed populations of T cells. Similar results were obtained for Th17 (ROR $\gamma \mathrm{T}$ versus CD4+IL17+) and Treg (Foxp3 versus CD $4^{+}$Foxp $^{+}$, Supplementary Figure 1).

IL-6 is a pleiotropic cytokine with important functions on different components of the immune system and is essential for Th17 differentiation [33]. IL- 6 combined with TGF- $\beta$ is necessary to induce the activation of ROR $\gamma \mathrm{t}$ and Th17 differentiation [34], whereas TGF- $\beta$ by itself induces Treg differentiation. Moreover, IL- 6 is able to inhibit TGF- $\beta$-induced Treg. Thus, the differentiation process of both cell types of cells is closely related $[35,36]$. An imbalance of Treg/Th17 is an important factor for triggering an autoimmune disease. We observed that MSCs injection in EAE mice, regardless of the time of administration, dramatically decreases IL- 6 which should promote the differentiation of Treg in vivo. Previous results from our laboratory showed that early injection of MSCs was able to induce a Treg response [32], which could explain the reduction of clinical score. Additionally, we observed a higher proportion of Treg (Foxp3/ROR $\gamma$ T ratio $>$ 1) in $\mathrm{EAE}+\mathrm{MSC}_{\text {onset }}$, where there was an early and prolonged decrease in circulating IL-6, providing additional evidence that MSCs-induced drop in IL-6 levels might promote Treg and decrease EAE. Furthermore, it has been described that IL-6 has important effect on the CNS and has been implicated in different diseases such as MS, Parkinson, and depression [37]. Also, it has been demonstrated that IL-6 increased the permeability of the $\mathrm{BBB}$ [38-40]. BBB consists of network of endothelial cells closely linked by tight junctions, forming a barrier with low permeability; therefore, the infiltration of lymphocytes into CNS is limited. The breakdown of $\mathrm{BBB}$ facilitates the infiltration of immune cells into the brain. Taken together, we propose that the inhibition of IL-6 allowed reestablishing, to some degree, the well-functioning 
of the $\mathrm{BBB}$; as a consequence, we observed a decreased number of infiltrating leukocytes across the BBB cell layer, despite the persisting differentiation and activation occurring in the lymph node or spleen as previously described.

Previous studies from our laboratory show that late additions of MSCs to $\mathrm{CD}^{+}$grown in polarized conditions towards Th1 or Th17 lineages inhibited Th1, but not Th17. This leads to a paradoxical increase of Th17 cells in specific MSCs-mediated immunosuppressive conditions [20]. Guo et al. also reported similar results. Fetal bone marrow-derived MSCs added to $\mathrm{CD} 4^{+} \mathrm{T}$ cells (MSCs/CD4 ${ }^{+} 1: 10$ ratio) inhibit Th1 but still promote the expansion of human Th17 cells [21]. Our in vivo results using the EAE model demonstrated no increase in ROR $\gamma \mathrm{T}$ mRNA levels in the brain, and we have previously reported that there is no increase of Th17 lymphocytes in lymph nodes of EAE mice treated with MSCs [32]. Cytokine analysis of EAE treated mice did not show an important increase of Th17 associated cytokines, IL-17A and IL-23; only an effect was observed on IL-17F at early time of MSCs injection. However, we observed the appearance of atypical signs of EAE, which is described to be associated with an increase in Th17 response, at late time of MSCs injection. An important decrease of Foxp3/ROR $\gamma \mathrm{T}$ and Foxp3/T-bet ratios $(<1)$ was detected in $\mathrm{EAE}+\mathrm{MSC}_{\text {peak }}$ and $\mathrm{EAE}+\mathrm{MSC}_{\text {late }}$ groups. Discrepancies could be explained by the timing of mRNA analysis, at the end point of experiment that could decrease sensitivity in detection of infiltrating Th17 into the brain after EAE score is decreased.

The appearance of atypical EAE when MSCs were injected after the onset of EAE surprised us greatly. In striking contrast, MSCs injected at the onset were highly effective. What are the reasons that may explain this difference? It is well known that the MSCs are "licensed" in vitro by proinflammatory cytokines such as IL- $1 \beta$, IFN- $\gamma$, and TNF$\alpha$ [41]. In view of our results, we favor the notion that the in vivo inflammatory microenvironment influences the immunosuppressive properties of MSCs when administered to EAE mice. MSCs injected at the onset of the disease effectively reduce both classic and atypical clinical scores. At the onset of EAE, cytokine levels were high. IL- 6 and Th1 and Th17 associated cytokines were 10 to 100 times higher than before EAE induction. We propose that the inflammatory microenvironment that MSCs find at the onset of EAE is efficient to induce an in vivo licensing of MSCs, enhancing the immunosuppressive capabilities of MSCs. It has been long time proposed that MSCs need the presence of inflammatory cytokines to become immunosuppressive. Initial findings with combinations of splenic T-cells and MSCs derived from knockout to either IFN- $\gamma$ or IFN- $\gamma$ R indicate IFN- $\gamma$ is essential to mediate this licensing process in MSCs. However, it also requires the combination of other proinflammatory cytokines to achieve a potent immunosuppressive phenotype $[42,43]$. We must consider that MSCs are exposed to a number of other stimuli, both activating and inhibitory signals. The balance of these signals ultimately determines the immunosuppressive potency of MSCs. In agreement, we have recently reported that MSCs are able to express a number of immunosuppressive mediators (NO2, TGF- $\beta 1$, Cox- 2 , and PGE2) after being cultured with supernatants from both Th1 and Th17. However, combination of two to three recombinant cytokines is imperative to reproduce this effect [32]. Interestingly, Luz-Crawford et al. demonstrated the important role of IL-17 as a "licenser" cytokine, which is consistent with the greater immunosuppressive capacity of MSCs injected at the onset, when the higher levels of Th17 associated cytokines (IL17A, IL-17F, and IL-23) were observed [32]. MSCs injected at the peak or at later stages of EAE were inefficient in reducing the clinical score and even induced the appearance of atypical signs of EAE. It is possible that the lower levels of proinflammatory cytokines were insufficient to properly license the MSCs. Moreover, high levels of IL-27 at the peak of the disease were observed, which remained elevated until day 30 of EAE (Supplementary Figure 2). The IL-27 antiinflammatory cytokine is associated with a regulatory profile [43], and the presence of this cytokine combined with a decrease in proinflammatory cytokines after the onset of EAE may be an important factor in determining the in vivo licensing of MSCs.

The final goal of this work is to call attention in the use of MSCs for the treatment of autoimmune diseases, especially to multiple sclerosis, which is characterized by heterogeneous clinical manifestations. MS patients show a diverse response to the treatment with immunomodulatory agents. Although MSCs have potent immunosuppressive capacity upon a licensing process in vitro, the administration of MSCs could induce unexpected effects. For example, in our EAE model, application of MSCs at the peak and at the time of EAE stabilization was able to induce the preferential appearance of atypical signs of the disease, probably by promoting an imbalance of Th1/Th17 versus Treg lymphocytes which could be associated with inefficient in vivo licensing process. Therefore, it is important to accurately assess the inflammatory status of patients prior to treatment with MSCs.

Further studies will allow us to better understand the complex process of MSC-mediated regulation of the immune system and to accurately predict how the patients will respond to MSC therapy.

\section{Conflict of Interests}

The authors report no conflict of interests regarding the publication of this paper.

\section{Authors' Contribution}

The study design was by Mónica Kurte and Flavio Carrión. Performed experiments were done by Mónica Kurte, Javiera Bravo-Alegría, Alexander Torres, Vania Carrasco, Cristina Ibáñez, Catalina Fernández-O’Ryan, and Rodrigo A. Fuentealba. Mónica Kurte, Javiera Bravo-Alegría, and Rodrigo A. Fuentealba analyzed the data. Claudia Riedel and Rodrigo A. Fuentealba contributed to analysis tools. Mónica Kurte, Flavio Carrión, Fernando E. Figueroa, Carlos E. Irarrázabal, and Ana María Vega-Letter were involved in writing the paper. All authors read and approved the final paper. 


\section{Acknowledgments}

The authors would like to thank Eduardo Albornoz for expert technical assistance with EAE model and Francisca Alcayaga for critical review of the paper. Also they sincerely thank X. Fernández, MF. Argaluza, and P. Luz-Crawford for the enormous technical assistance. This work was supported by Fondo Nacional de Desarrollo Científico y Tecnológico (FONDECYT) $\mathrm{N}^{\circ} 1130444$ and Grants of Universidad de los Andes FAI-MED-002-2010, FAI-PUENTE-03-2011, and FAIMED-049-2012.

\section{References}

[1] S. P. Bruder, D. J. Fink, and A. I. Caplan, "Mesenchymal stem cells in bone development, bone repair, and skeletal regeneration therapy," Journal of Cellular Biochemistry, vol. 56, no. 3, pp. 283-294, 1994.

[2] M. Monti, C. Perotti, C. del Fante, M. Cervio, and C. A. Redi, "Stem cells: sources and therapies," Biological Research, vol. 45, no. 3, pp. 207-214, 2012.

[3] M. F. Pittenger, A. M. Mackay, S. C. Beck et al., "Multilineage potential of adult human mesenchymal stem cells," Science, vol. 284, no. 5411, pp. 143-147, 1999.

[4] A. I. Caplan and S. P. Bruder, "Mesenchymal stem cells: building blocks for molecular medicine in the 21st century," Trends in Molecular Medicine, vol. 7, no. 6, pp. 259-264, 2001.

[5] A. Bartholomew, C. Sturgeon, M. Siatskas et al., "Mesenchymal stem cells suppress lymphocyte proliferation in vitro and prolong skin graft survival in vivo," Experimental Hematology, vol. 30, no. 1, pp. 42-48, 2002.

[6] M. di Nicola, C. Carlo-Stella, M. Magni et al., "Human bone marrow stromal cells suppress T-lymphocyte proliferation induced by cellular or nonspecific mitogenic stimuli," Blood, vol. 99, no. 10, pp. 3838-3843, 2002.

[7] F. Djouad, P. Plence, C. Bony et al., "Immunosuppressive effect of mesenchymal stem cells favors tumor growth in allogeneic animals," Blood, vol. 102, no. 10, pp. 3837-3844, 2003.

[8] A. Uccelli, L. Moretta, and V. Pistoia, "Immunoregulatory function of mesenchymal stem cells," European Journal of Immunology, vol. 36, no. 10, pp. 2566-2573, 2006.

[9] A. Tyndall, U. A. Walker, A. Cope et al., "Immunomodulatory properties of mesenchymal stem cells: a review based on an interdisciplinary meeting held at the Kennedy Institute of Rheumatology Division, London, UK, 31 October 2005," Arthritis Research \& Therapy, vol. 9, no. 1, pp. 301-310, 2007.

[10] M. Shi, Z.-W. Liu, and F.-S. Wang, "Immunomodulatory properties and therapeutic application of mesenchymal stem cells," Clinical and Experimental Immunology, vol. 164, no. 1, pp. 1-8, 2011.

[11] F. E. Figueroa, F. Carrión, S. Villanueva, and M. Khoury, "Mesenchymal stem cell treatment for autoimmune diseases: a critical review," Biological Research, vol. 45, no. 3, pp. 269-277, 2012.

[12] F. Carrion, E. Nova, C. Ruiz et al., "Autologous mesenchymal stem cell treatment increased T regulatory cells with no effect on disease activity in two systemic lupus erythematosus patients," Lupus, vol. 19, no. 3, pp. 317-322, 2010.
[13] S. T. Lee, J. H. Jang, J.-W. Cheong et al., “Treatment of high-risk acute myelogenous leukaemia by myeloablative chemoradiotherapy followed by co-infusion of T cell-depleted haematopoietic stem cells and culture-expanded marrow mesenchymal stem cells from a related donor with one fully mismatched human leucocyte antigen haplotype," British Journal of Haematology, vol. 118, no. 4, pp. 1128-1131, 2002.

[14] E. Zappia, S. Casazza, E. Pedemonte et al., "Mesenchymal stem cells ameliorate experimental autoimmune encephalomyelitis inducing T-cell anergy," Blood, vol. 106, no. 5, pp. 1755-1761, 2005.

[15] X.-J. Liu, J.-F. Zhang, B. Sun et al., "Reciprocal effect of mesenchymal stem cell on experimental autoimmune encephalomyelitis is mediated by transforming growth factor- $\beta$ and interleukin-6," Clinical and Experimental Immunology, vol. 158, no. 1, pp. 37-44, 2009.

[16] F. Djouad, V. Fritz, F. Apparailly et al., "Reversal of the immunosuppressive properties of mesenchymal stem cells by tumor necrosis factor $\alpha$ in collagen-induced arthritis," Arthritis and Rheumatism, vol. 52, no. 5, pp. 1595-1603, 2005.

[17] M. A. González, E. González-Rey, L. Rico, D. Büscher, and M. Delgado, "Treatment of experimental arthritis by inducing immune tolerance with human adipose-derived mesenchymal stem cells," Arthritis and Rheumatism, vol. 60, no. 4, pp. 10061019, 2009.

[18] K. Zhou, H. Zhang, O. Jin et al., “Transplantation of human bone marrow mesenchymal stem cell ameliorates the autoimmune pathogenesis in MRL/lpr mice," Cellular and Molecular Immunology, vol. 5, no. 6, pp. 417-424, 2008.

[19] K. Savvatis, S. van Linthout, K. Miteva et al., "Mesenchymal stromal cells but not cardiac fibroblasts exert beneficial systemic immunomodulatory effects in experimental myocarditis," PLoS ONE, vol. 7, no. 7, Article ID e41047, 2012.

[20] F. Carrión, E. Nova, P. Luz, F. Apablaza, and F. Figueroa, "Opposing effect of mesenchymal stem cells on Th1 and Th17 cell polarization according to the state of $\mathrm{CD}^{+} \mathrm{T}$ cell activation," Immunology Letters, vol. 135, no. 1-2, pp. 10-16, 2011.

[21] Z. Guo, C. Zheng, Z. Chen et al., "Fetal BM-derived mesenchymal stem cells promote the expansion of human Th17 cells, but inhibit the production of Th1 cells," European Journal of Immunology, vol. 39, no. 10, pp. 2840-2849, 2009.

[22] D. E. Goodkin, W. D. Rooney, R. Sloan et al., "A serial study of new MS lesions and the white matter from which they arise," Neurology, vol. 51, no. 6, pp. 1689-1697, 1998.

[23] C. Hedegaard, M. Krakauer, K. Bendtzen et al., “T helper cell type 1 (Th1), Th2 and Th17 responses to myelin basic protein and disease activity in multiples sclerosis," Annals of Neurology, vol. 66, pp. 390-402, 2009.

[24] H. Kebir, I. Ifergan, J. I. Alvarez et al., "Preferential recruitment of interferon- $\gamma$-expressing TH17 cells in multiple sclerosis," Annals of Neurology, vol. 66, no. 3, pp. 390-402, 2009.

[25] P. J. Darlington, M.-N. Boivin, C. Renoux et al., "Reciprocal Th1 and Th17 regulation by mesenchymal stem cells: implication for multiple sclerosis," Annals of Neurology, vol. 68, no. 4, pp. 540545, 2010.

[26] T. Korn, J. Reddy, W. Gao et al., "Myelin-specific regulatory T cells accumulate in the CNS but fail to control autoimmune inflammation," Nature Medicine, vol. 13, no. 4, pp. 423-431, 2007.

[27] R. A. O'Connor and S. M. Anderton, "Foxp3 ${ }^{+}$regulatory T cells in the control of experimental CNS autoimmune disease," Journal of Neuroimmunology, vol. 193, no. 1-2, pp. 1-11, 2008. 
[28] I. M. Stromnes, L. M. Cerretti, D. Liggitt, R. A. Harris, and J. M. Goverman, "Differential regulation of central nervous system autoimmunity by $\mathrm{T}_{H} 1$ and $\mathrm{T}_{H} 17$ cells," Nature Medicine, vol. 14, no. 3, pp. 337-342, 2008.

[29] H. S. Domingues, M. Mues, H. Lassmann, H. Wekerle, and G. Krishnamoorthy, "Functional and pathogenic differences of Th1 and Th17 cells in experimental autoimmune encephalomyelitis," PLoS ONE, vol. 5, no. 11, Article ID e15531, 2010.

[30] K. J. Livak and T. D. Schmittgen, "Analysis of relative gene expression data using real-time quantitative PCR and the 2-(Delta Delta C(T)) CT method," Methods, vol. 25, no. 4, pp. 402408, 2001.

[31] M. Dominici, K. Le Blanc, I. Mueller et al., "Minimal criteria for defining multipotent mesenchymal stromal cells. The International Society for Cellular Therapy position statement," Cytotherapy, vol. 8, no. 4, pp. 315-317, 2006.

[32] P. Luz-Crawford, M. Kurte, J. Bravo-Alegría et al., "Mesenchymal stem cells generate a $\mathrm{CD} 4{ }^{+} \mathrm{CD} 25^{+}$Foxp $3^{+}$regulatory $\mathrm{T}$ cell population during the differentiation process of Th1 and Th17 cells," Stem Cell Research and Therapy, vol. 4, no. 3, article 65, 2013.

[33] B. Stockinger, M. Veldhoen, and B. Martin, “Th17 T cells: linking innate and adaptive immunity," Seminars in Immunology, vol. 19, no. 6, pp. 353-361, 2007.

[34] A. Laurence and J. J. O'Shea, " $\mathrm{T}_{H}-17$ differentiation: of mice and men," Nature Immunology, vol. 8, no. 9, pp. 903-905, 2007.

[35] S. Ikeda, S. Saijo, M. A. Murayama, K. Shimizu, A. Akitsu, and Y. Iwakura, "Excess IL-1 signaling enhances the development of Th17 cells by downregulating TGF-beta-induced Foxp3 expression," Journal of Immunology, vol. 192, no. 4, pp. 14491458, 2014.

[36] J. Zhu and W. E. Paul, "Heterogeneity and plasticity of T helper cells," Cell Research, vol. 20, no. 1, pp. 4-12, 2010.

[37] A. Spooren, K. Kolmus, G. Laureys et al., "Interleukin-6, a mental cytokine," Brain Research Reviews, vol. 67, no. 1-2, pp. 157-183, 2011.

[38] J. Labus, S. Häckel, L. Lucka, and K. Danker, "Interleukin-1 $\beta$ induces an inflammatory response and the breakdown of the endothelial cell layer in an improved human THBMEC-based in vitro blood-brain barrier model," Journal of Neuroscience Methods, vol. 228, pp. 35-45, 2014.

[39] A. Eskilsson, E. Mirrasekhian, S. Dufour, M. Schwaninger, D. Engblom, and A. Blomqvist, "Immune-induced fever is mediated by IL-6 receptors on brain endothelial cells coupled to STAT3-dependent induction of brain endothelial prostaglandin synthesis," Journal of Neuroscience, vol. 34, no. 48, pp. 1595715961, 2014.

[40] K. D. Rochfort, L. E. Collins, R. P. Murphy, and P. M. Cummins, "Downregulation of blood-brain barrier phenotype by proinflammatory cytokines involves NADPH oxidase-dependent ROS generation: consequences for interendothelial adherens and tight junctions," PLoS ONE, vol. 9, no. 7, Article ID e101815, 2014.

[41] M. Krampera, "Mesenchymal stromal cell licensing: a multistep process," Leukemia, vol. 25, no. 9, pp. 1408-1414, 2011.

[42] G. Ren, L. Zhang, X. Zhao et al., "Mesenchymal stem cellmediated immunosuppression occurs via concerted action of chemokines and nitric oxide," Cell Stem Cell, vol. 2, no. 2, pp. 141-150, 2008.

[43] C. A. Hunter and R. Kastelein, "Interleukin-27: balancing protective and pathological immunity," Immunity, vol. 37, no. 6, pp. 960-969, 2012. 

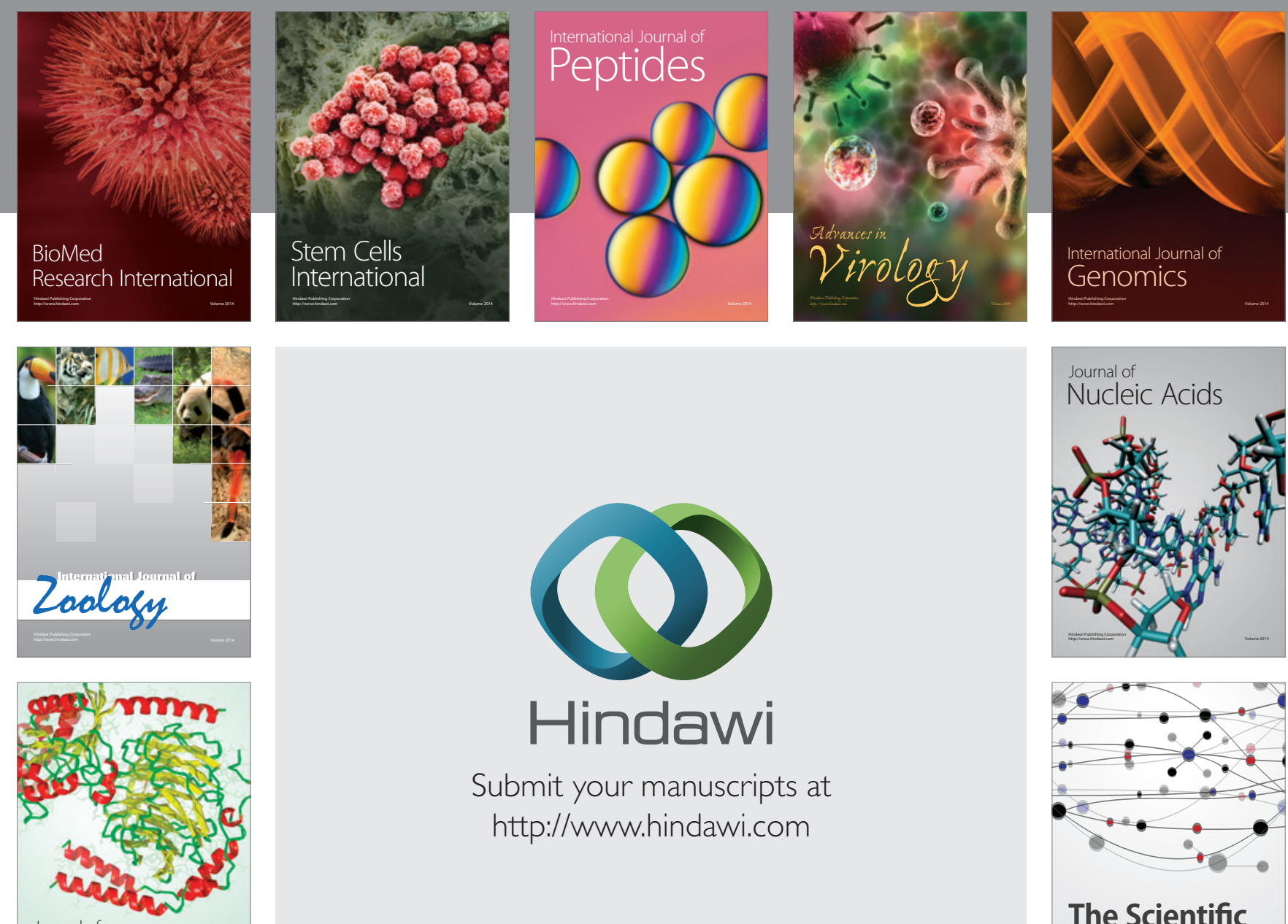

Submit your manuscripts at

http://www.hindawi.com

Journal of
Signal Transduction
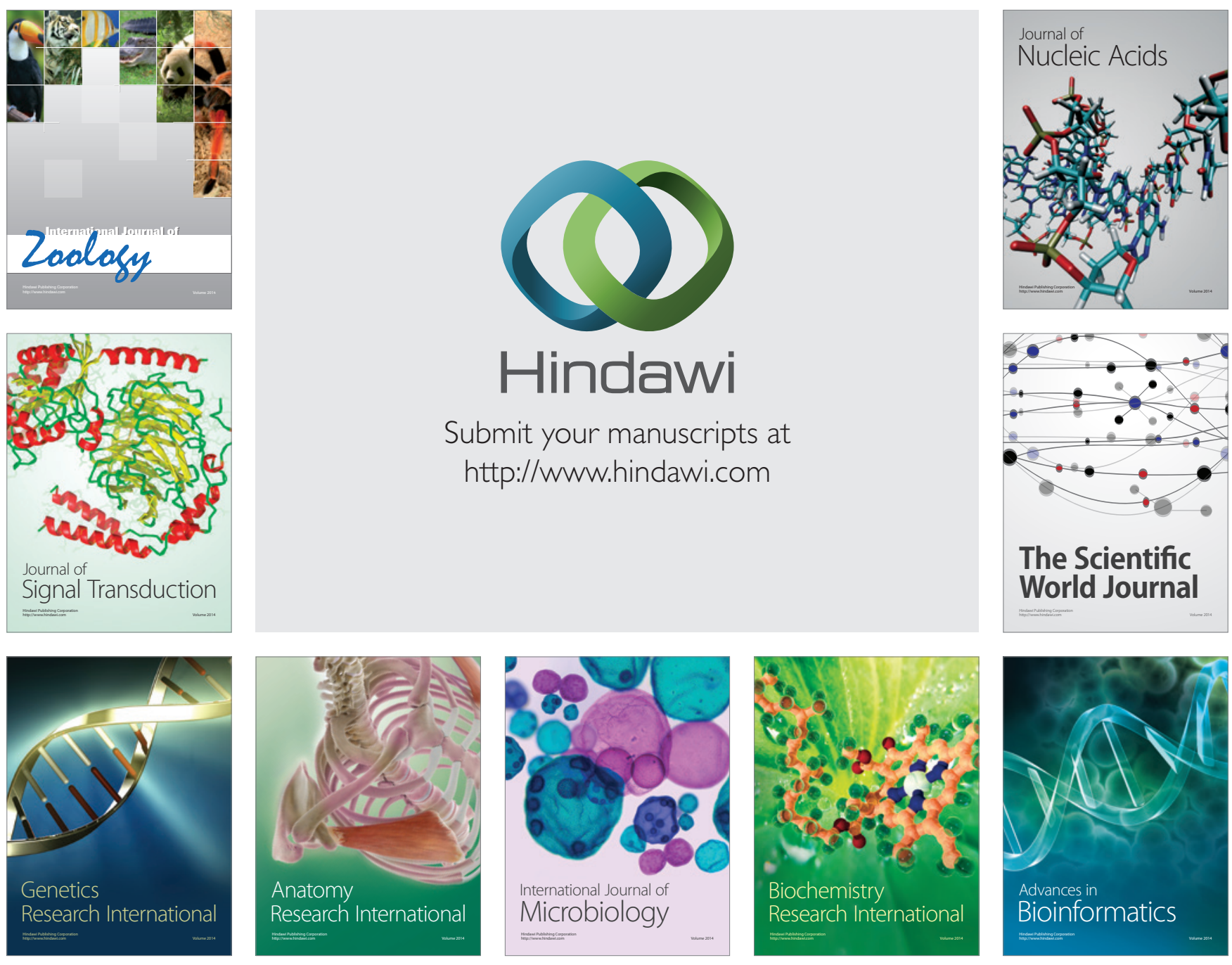

The Scientific World Journal
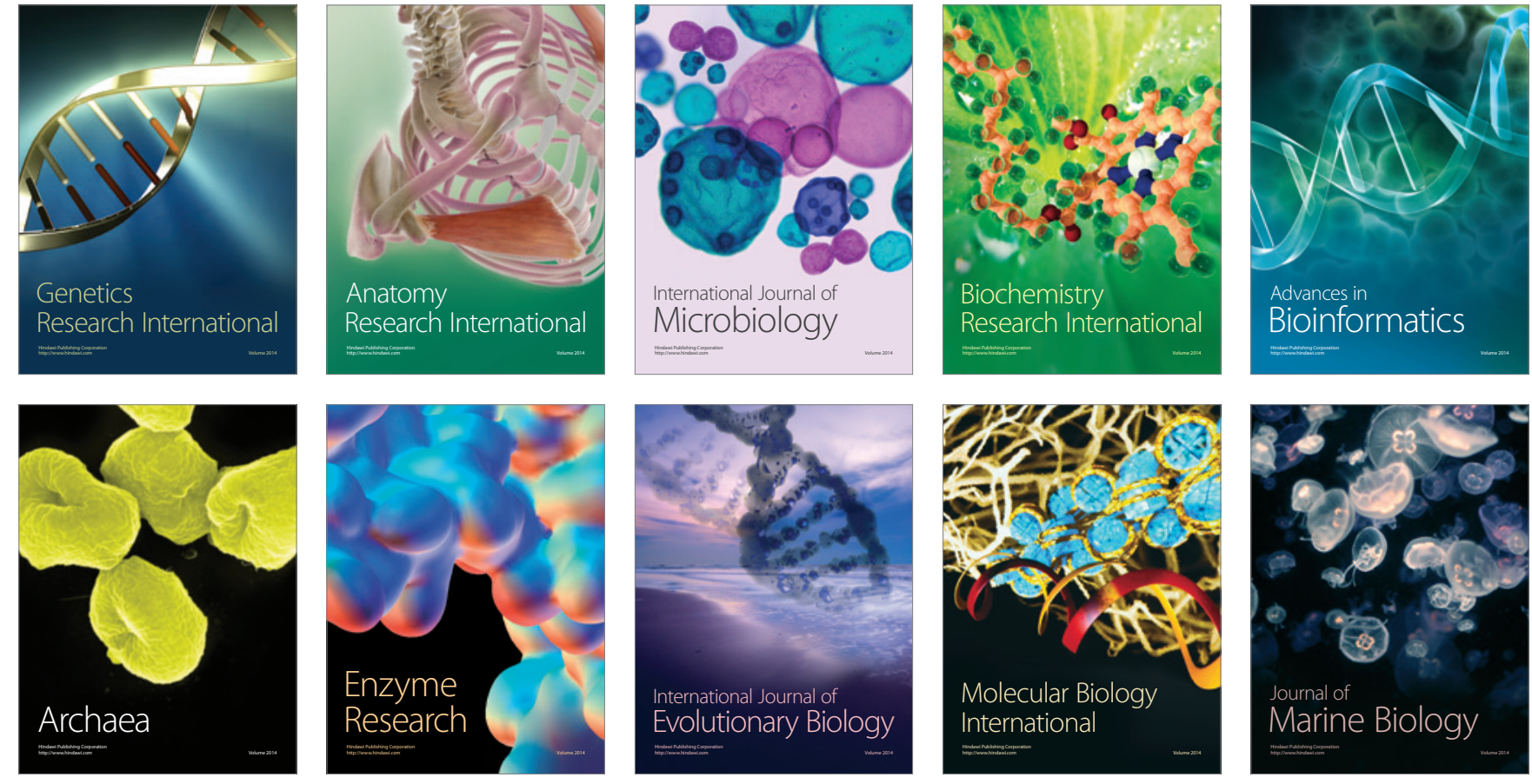